\title{
Finite-Time Nonfragile Synchronization of Stochastic Complex Dynamical Networks with Semi-Markov Switching Outer Coupling
}

\author{
Rathinasamy Sakthivel $\mathbb{D}^{1,2}$ Ramalingam Sakthivel, ${ }^{3}$ Boomipalagan Kaviarasan, ${ }^{3}$ \\ Chao Wang, ${ }^{4}$ and Yong-Ki Ma $\oplus^{5}$ \\ ${ }^{1}$ Department of Mathematics, Bharathiar University, Coimbatore 641046, India \\ ${ }^{2}$ Department of Mathematics, Sungkyunkwan University, Suwon 440-746, Republic of Korea \\ ${ }^{3}$ Department of Mathematics, Anna University Regional Campus, Coimbatore 641046, India \\ ${ }^{4}$ Department of Mathematics, Yunnan University, Kunming, Yunnan 650091, China \\ ${ }^{5}$ Department of Applied Mathematics, Kongju National University, Chungcheongnam-do 32588, Republic of Korea
}

Correspondence should be addressed to Yong-Ki Ma; ykma@kongju.ac.kr

Received 13 July 2017; Accepted 14 December 2017; Published 16 January 2018

Academic Editor: Hiroki Sayama

Copyright (C) 2018 Rathinasamy Sakthivel et al. This is an open access article distributed under the Creative Commons Attribution License, which permits unrestricted use, distribution, and reproduction in any medium, provided the original work is properly cited.

\begin{abstract}
The problem of robust nonfragile synchronization is investigated in this paper for a class of complex dynamical networks subject to semi-Markov jumping outer coupling, time-varying coupling delay, randomly occurring gain variation, and stochastic noise over a desired finite-time interval. In particular, the network topology is assumed to follow a semi-Markov process such that it may switch from one to another at different instants. In this paper, the random gain variation is represented by a stochastic variable that is assumed to satisfy the Bernoulli distribution with white sequences. Based on these hypotheses and the Lyapunov-Krasovskii stability theory, a new finite-time stochastic synchronization criterion is established for the considered network in terms of linear matrix inequalities. Moreover, the control design parameters that guarantee the required criterion are computed by solving a set of linear matrix inequality constraints. An illustrative example is finally given to show the effectiveness and advantages of the developed analytical results.
\end{abstract}

\section{Introduction}

During the past twenty years, the investigation of complex dynamical networks (CDNs) that consist of a huge number of interacting dynamical nodes has received a great deal of attention from various science and engineering areas, such as social networks, ecological prey-predator networks, protein networks, power grids, and ecosystems $[1,2]$. It should be mentioned that the analysis of dynamical behaviors of CDNs has become a hot research topic in recent years. Among many dynamical behaviors, synchronization phenomenon is the most important behavior and several interesting and efficient methodologies have been developed in the literature to solve the synchronization problem of various kinds of CDNs; for instance, see [3-7]. In [4], the problem of inner synchronization of a CDN has been investigated by considering two different types of guaranteed cost dynamic feedback controllers, where the control gains corresponding to two feedback controllers have different dimensions subject to the topological structure of the CDN. In [5], the problem of outer synchronization between two hybrid-coupled delayed dynamical networks has been discussed by using the aperiodically adaptive intermittent pinning control, where a simple and elegant pinned-node selection scheme is proposed to achieve the required result. It is worth mentioning that the $\mathrm{CDN}$ representing real-time systems is generally affected by external noise factors or stochastic disturbances [8]. Thus, the consideration of external noise factors in the study of synchronization of CDNs is of great importance in the viewpoints of both theoretical and practical. By taking this 
fact into account, in recent years, research communities have eagerly investigated the problems of synchronization of CDNs with external stochastic disturbances, see for example [9-12]. Sakthivel et al. [10] presented some sufficient conditions that ensure the synchronization and solve the state estimation problem of discrete-time stochastic complex networks in the presence of uncertain inner couplings, where the interval matrix approach is employed to characterize the uncertainties encountered in the inner coupling terms. $\mathrm{Li}$ et al. [11] developed a new synchronization criterion for a class of discrete-time stochastic complex networks subject to partial mixed impulsive effects, where by using the Lyapunov stability theory and the variation of parameters formula, the required criterion is obtained.

It should be pointed out that the interconnection topology among the nodes of CDNs plays a significant role in the study of synchronization problem. In the existing literature, there have been two reported kinds of interconnection topologies, which are constant/fixed topology [13] and timevarying topology [14]. In [14], it has been illustrated that the time-varying interconnection topology is more general than the fixed one. Nevertheless, in most of the real networks, the connectivity of the network topology might be unfixed or randomly changing due to new creation or link failures. In order to tackle these types of issues, it is more appropriate to model the CDNs with randomly switching topologies that are governed by a Markov process. Based on this scenario and following the seminal works reported in $[15,16]$, some interesting and significant results about synchronization of CDNs with Markov jump topologies have been discussed; see [17-19]. Specifically, in [18], the problem of nonfragile synchronization for a class of discrete-time complex networks subject to Markov jumping switching topology has been investigated in a unified framework that includes the nonfragile $\mathscr{H}_{\infty}$ synchronization control and nonfragile $l_{2}-l_{\infty}$ synchronization control problem as its special cases. However, it is worth pointing out that the aforementioned papers have considered constant transition rates in the Markov process. It should be mentioned that the Markov process might consist of time-varying transition rates when modeling practical systems. Such kind of process is known as semi-Markov process and few interesting results regarding semi-Markov jump systems have been addressed in recent literature [20-23]. Interestingly, in [21], by employing the supplementary variable technique and plant transformation, the state estimation and sliding mode control problems have been investigated for semi-Markovian jump systems in the presence of mismatched uncertainties. Apparently, semi-Markov jump systems are comparatively more general than the traditional Markov jump systems [24]. Following the aforementioned seminal works, the concept of semiMarkov process has further been employed in the network topology to obtain the synchronization criteria for CDNs (see $[25,26]$ and the references therein). However, only very few results about the synchronization of CDNs with semi-Markov jump topology have been reported in the literature, which stimulates us to do this present work.

It is worth mentioning that most of the available controller design approaches have a predominant assumption that the designed controller can be implemented accurately. But in some real situations, such an assumption is not always true as the controllers are often very sensitive or fragile to their parameters' variations. Furthermore, it should be noted that a small perturbation in controllers may lead to undesirable oscillatory behavior or even instability [27]. Hence, it is desirable as well as necessary to ensure the insensitivity of the controller to certain parameter perturbations without loss of the robust stability and thus, the investigation of nonfragile or resilient controller design that has been capable of tolerating some level of controller parameter gain variations has been enormously increased in recent years [28-31]. To mention a few, in [28], a robust resilient control problem of discretetime Markov jump nonlinear systems has been solved by employing the linear matrix inequality and stochastic analysis techniques; in [29], based on the dissipative theory and the event-triggered sampling scheme, the nonfragile control design problem for a class of network-based singular systems with input time-varying delay and external disturbances has been addressed. Therefore, it is reasonable to consider the nonfragile control design in the study of synchronization of CDNs. It is noteworthy that only few research papers regarding the nonfragile control design for achieving the synchronization of CDNs have been published; see [32, 33]. On the other hand, it is worth mentioning that most of the existing results based on the classical control theory dealt with the asymptotic property of control system trajectories over an infinite-time interval and did not possess any restriction to the system states. But in many practical problems, it is required that the described system state does not exceed a certain bound during a fixed finite-time interval [34]. According to this fact, a great number of interesting results on finite-time control design have been proposed for the synchronization of various CDNs; for instance, see [35-38]. To the best of our knowledge, however, the problem of robust nonfragile synchronization has not been fully investigated for a class of CDNs over a prescribed finite-time interval.

Motivated by the above analysis, in this paper, we focus on the finite-time nonfragile synchronization problem is investigated for a class of CDNs subject to semi-Markov jump topology and stochastic noises. More precisely, a new delaydependent sufficient condition under which the considered CDNs are synchronized to the target network within a given finite-time interval is developed in terms of linear matrix inequalities by utilizing the Lyapunov stability theory and the stochastic analysis techniques. Subsequently, based on the developed condition, a design algorithm of the proposed nonfragile state feedback controller that can ensure the finitetime stochastic synchronization of the addressed network is presented. Eventually, a numerical example is shown to illustrate the effectiveness of the proposed theoretical results.

The rest of this paper is organized as follows: in Section 2, the problem formulation of the network model under study and the preliminaries required to obtain the main results are given. The finite-time stochastic synchronization criterion for the considered network model is presented in Section 3. A numerical example and its simulations are provided in Section 4. Conclusion of this paper is given in Section 5 . 


\section{Problem Formulation and Preliminaries}

In this paper, we consider a class of complex dynamical networks (CDNs) with semi-Markov jump outer coupling and stochastic noise, which consists of $N$ identical nodes and is defined over the Wiener process probability space $(\Omega, \mathscr{F}, \mathbb{P})$, where $\Omega$ is the sample space, $\mathscr{F}$ is the algebra of events and $\mathbb{P}$ is the probability measure defined on $\mathscr{F}$. Such a network model can be described in the following form:

$$
\begin{aligned}
& d x_{i}(t)=\left[A x_{i}(t)+f\left(t, x_{i}(t)\right)\right. \\
& \left.+\lambda \sum_{j=1}^{N} T_{i j}(\sigma(t)) \Lambda x_{j}(t-\tau(t))+u_{i}(t)\right] d t \\
& +\rho\left(t, x_{i}(t), x_{i}(t-\tau(t))\right) d w(t), \\
& \quad x_{i}(t)=\phi_{i}(t), \forall t \in\left[-\tau_{2}, 0\right], i=1,2, \ldots, N,
\end{aligned}
$$

where $x_{i}(t) \in \mathbb{R}^{n}$ denotes the state vector of the $i$ th node; $A$ is a known real constant matrix with suitable dimension; $f(\cdot, \cdot) \in \mathbb{R}^{n}$ represents a nonlinear vector-valued function; the constant $\lambda>0$ is the coupling strength of network; $T_{i j}(\sigma(t))$ are the elements of the outer coupling matrix $T(\sigma(t))$ which describes the network topological structure and is assumed to follow a semi-Markov process $\sigma(t)$ which to be defined later. In particular, $T_{i j}(\sigma(t))$ is defined as follows: if there exists a connection between node $i$ and node $j$, then $T_{i j}(\sigma(t))>0$; otherwise, $T_{i j}(\sigma(t))=0$. Further, the diagonal elements of the outer coupling matrix are given as follows: $T_{i i}(\sigma(t))=-\sum_{j=1, j \neq i}^{N} T_{i j}(\sigma(t)) ; \Lambda$ represents the inner coupling matrix and is a positive diagonal matrix with appropriate dimension; $u_{i}(t) \in \mathbb{R}^{n}$ is the control input of the $i$ th node which to be defined later; the function $\rho(\cdot, \cdot, \cdot): \mathbb{R} \times$ $\mathbb{R}^{n} \times \mathbb{R}^{n} \rightarrow \mathbb{R}^{n}$ is the noise intensity vector-valued function; $w(t)$ is a 1-dimensional Brownian motion defined on the probability space $(\Omega, \mathscr{F}, \mathbb{P})$ with $\mathbb{E}\{w(t)\}=0, \mathbb{E}\left\{w^{2}(t)\right\}=1$ and $\mathbb{E}\{w(s) w(t)\}=0$ for $s \neq t$, where $\mathbb{E}$ is the mathematical expectation; $\tau(t)$ is the time-varying delay function satisfying $0 \leq \tau_{1} \leq \tau(t) \leq \tau_{2}<\infty$ and $\dot{\tau}(t) \leq \mu<1$, where $\tau_{1}$, $\tau_{2}$, and $\mu$ are known scalars; and $\phi_{i}(t)$ denotes the initial value of the $i$ th node's state and is assumed to be a continuous vector-valued function.

Now, let us define the semi-Markov jump process of the outer coupling matrix. The process $\{\sigma(t), t \geq 0\}$ is a continuous-time homogeneous semi-Markov process with right continuous trajectories and takes values in a finite set $\mathbb{S}=\{1,2, \ldots, \mathscr{N}\}$. More precisely, $\sigma(t)$ is associated with the transition probability matrix $\Pi=\left[\pi_{p q}(h)\right]_{\mathscr{N} \times \mathcal{N}}$ which is given by the following transition rates:

$$
\begin{aligned}
\operatorname{Prob}\{\sigma(t+h)=q \mid \sigma(t)=p\} \\
\quad= \begin{cases}\pi_{p q}(h) h+o(h), & \text { if } p \neq q, \\
1+\pi_{p p}(h) h+o(h), & \text { if } p=q,\end{cases}
\end{aligned}
$$

where $h>0$ is the sojourn time, $\lim _{h \rightarrow 0}(o(h) / h)=0$ and $\pi_{p q}(h) \geq 0$ for $p \neq q$ is the transition rate from mode $p$ at time $t$ to mode $q$ at time $t+h$ and $\pi_{p p}(h)=-\sum_{q=1, q \neq p}^{\mathcal{N}} \pi_{p q}(h)$. For notational simplicity, we hereafter denote the semi-Markov process parameter $\sigma(t)$ by $p$. For example, $T(\sigma(t))$ is denoted by $T_{p}$.

To synchronize all the $N$ identical nodes in the network (1) to a common value, let us define the synchronization error vector as $e_{i}(t)=x_{i}(t)-s(t)$, where $s(t) \in \mathbb{R}^{n}$ is the state vector of the unforced isolated node that can be expressed as $d s(t)=[A s(t)+f(t, s(t))] d t$ and is assumed to be noise-free, that is, $\rho(t, s(t), s(t-\tau(t)))=0$. Based on this error vector, we now choose a robust state feedback controller to achieve the synchronization of network (1), which is insensitive to the uncertain perturbations or gain fluctuations and of the form:

$$
u_{i}(t)=\left(K_{p}+\alpha(t) \Delta K_{p}(t)\right) e_{i}(t), \quad i=1,2, \ldots, N,
$$

where $K_{p}$ is the feedback controller gain matrix that is to be determined in the forthcoming section, $\Delta K_{p}(t)$ is a timevarying matrix representing the controller gain fluctuations, and $\alpha(t)$ is a stochastic variable describing the randomly occurring controller gain fluctuations. It is here assumed that $\Delta K_{p}(t)$ takes the form $\Delta K_{p}(t)=M_{p} \Gamma_{p}(t) N_{p}$, where $M_{p}$ and $N_{p}$ are known real constant matrices and $\Gamma_{\mathrm{p}}(t)$ is an unknown time-varying matrix satisfying $\Gamma_{p}^{T}(t) \Gamma_{p}(t) \leq I$. Further, it is assumed that the stochastic variable $\alpha(t)$ obeys the Bernoulli distribution with the following probability rules: (i) $\operatorname{Prob}\{\alpha(t)=1\}=\mathbb{E}\{\alpha(t)\}=\bar{\alpha}$ and (ii) Prob $\{\alpha(t)=$ $0\}=1-\mathbb{E}\{\alpha(t)\}=1-\bar{\alpha}$, where $\bar{\alpha} \in[0,1]$.

Then, by using (1) and (3), the closed-loop form of the error system can be obtained as follows:

$$
\begin{aligned}
& d e_{i}(t)=\left[\left(A+K_{p}+\alpha(t) \Delta K_{p}(t)\right) e_{i}(t)+g\left(t, e_{i}(t)\right)\right. \\
& \left.+\lambda \sum_{j=1}^{N} T_{i j p} \Lambda e_{j}(t-\tau(t))\right] d t \\
& +\hat{\rho}\left(t, e_{i}(t), e_{i}(t-\tau(t))\right) d w(t), \\
& \quad p \in \mathbb{S}, i=1,2, \ldots, N,
\end{aligned}
$$

where $g\left(t, e_{i}(t)\right)=f\left(t, x_{i}(t)\right)-f(t, s(t))$ and $\hat{\rho}\left(t, e_{i}(t), e_{i}(t-\right.$ $\tau(t)))=\rho\left(t, x_{i}(t), x_{i}(t-\tau(t))\right)-\rho(t, s(t), s(t-\tau(t)))$. By using the Kronecker product properties and mathematical manipulations, the error system (4) can be written in the following compact form:

$$
\begin{aligned}
d e & (t)=\left[\left(\left(I_{N} \otimes A\right)+\left(I_{N} \otimes K_{p}\right)\right.\right. \\
+ & \bar{\alpha}\left(I_{N} \otimes M_{p} \Gamma_{p}(t) N_{p}\right) \\
+ & \left.(\alpha(t)-\bar{\alpha})\left(I_{N} \otimes M_{p} \Gamma_{p}(t) N_{p}\right)\right) e(t)+G(t, e(t)) \\
& \left.+\lambda\left(T_{p} \otimes \Lambda\right) e(t-\tau(t))\right] d t+\hat{\rho}(t, e(t), e(t \\
& -\tau(t))) d w(t)
\end{aligned}
$$


where $e(t)=\left[e_{1}^{T}(t), e_{2}^{T}(t), \ldots, e_{N}^{T}(t)\right]^{T}, G(t, e(t))=\left[g^{T}(t\right.$, $\left.\left.e_{1}(t)\right), g^{T}\left(t, e_{2}(t)\right), \ldots, g^{T}\left(t, e_{N}(t)\right)\right]^{T}$, and $\hat{\rho}(t, e(t), \quad e(t-$ $\tau(t)))=\left[\hat{\rho}\left(t, e_{1}(t), e_{1}(t-\tau(t))\right), \widehat{\rho}\left(t, e_{2}(t), e_{2}(t-\tau(t))\right), \ldots\right.$, $\left.\hat{\rho}\left(t, e_{N}(t), e_{N}(t-\tau(t))\right)\right]^{T}$.

In order to develop the main results, the following assumptions and definition are required.

Assumption 1. For the nonlinear function $f(\cdot, \cdot)$, there exists a known real constant matrix $\mathscr{G}$ such that $\left\|f\left(t, x_{i}(t)\right)\right\| \leq$ $\mathscr{G}\left\|x_{i}(t)\right\|$ for any $x_{i}(t) \in \mathbb{R}^{n}$.

Assumption 2. The noise intensity function $\rho(\cdot, \cdot, \cdot): \mathbb{R}^{+} \times$ $\mathbb{R}^{n} \times \mathbb{R}^{n} \rightarrow \mathbb{R}^{n}$ is uniformly Lipschitz continuous in terms of the following inequality of trace inner product: $\operatorname{trace}\left\{\rho^{T}\left(t, x_{i}(t), x_{i}(t-\tau(t))\right) \rho\left(t, x_{i}(t), x_{i}(t-\tau(t))\right)\right\} \leq$ $\eta_{i} x_{i}^{T}(t) x_{i}(t)+\zeta_{i} x_{i}^{T}(t-\tau(t)) x_{i}(t-\tau(t))$, where $\eta_{i}$ and $\zeta_{i}(i=$ $1,2, \ldots, N)$ are known nonnegative constants.

Assumption 3. For each $p \in \mathbb{S}$, all the real parts of eigenvalues of $T(\sigma(t))$ are negative except an eigenvalue 0 with multiplicity 1 , which means that the reverse of the graph generated by the matrix $T(\sigma(t))$ contains a rooted spanning directed tree for every $p \in \mathbb{S}$.

Definition 4 (see [34]). The considered network (1) is said to be stochastically synchronized in finite-time with respect to $\left(c_{1}, c_{2}, \tau_{2}, T^{*}, Z_{p}\right)$ if there exist positive definite matrix $Z_{p}(p \in \mathbb{S})$ and positive constants $T^{*}, c_{1}, c_{2}$ with $c_{2}>c_{1}$ such that the following condition holds:

$$
\begin{aligned}
\mathbb{E}\left\{x^{T}\left(t_{0}\right)\left(I_{N} \otimes Z_{p}\right) x\left(t_{0}\right)\right\} & \leq c_{1} \\
\Longrightarrow \mathbb{E}\left\{x^{T}(t)\left(I_{N} \otimes Z_{p}\right) x(t)\right\} & <c_{2}, \\
t_{0} & \in\left[-\tau_{2}, 0\right], t \in\left[0, T^{*}\right] .
\end{aligned}
$$

\section{Main Results}

Based on the Lyapunov-Krasovskii stability theory, this section aims to develop a new set of delay-dependent sufficient conditions that can guarantee the stochastic synchronization of the considered network model (1) over a finite-time interval. Moreover, based on these conditions, a design of the robust nonfragile state feedback control (3) for the network model under consideration is provided in terms of linear matrix inequalities (LMIs).

Theorem 5. Consider the network model (1) with Assumptions 1-3. For given positive scalars $c_{1}, c_{2}, T^{*}, \delta, \mu, \lambda, \bar{\alpha} \in[0,1]$, $\tau_{1}, \tau_{2}$, symmetric matrix $Z_{p}(p \in \mathbb{S})$, and diagonal matrices $R_{3}, R_{4}$, the considered network (1) is stochastically synchronized in finite-time under the nonfragile controller (3), if there exist symmetric matrices $P_{p}>0(p \in \mathbb{S}), Q_{l}>0(l=1,2,3)$, $R_{v}>0(v=1,2)$ and positive scalars $\kappa_{p}(p \in \mathbb{S}), \epsilon_{1}$ such that the following matrix inequalities hold:

$$
P_{p}-\kappa_{p} I_{N}<0, \quad p \in \mathbb{S},
$$

$$
\begin{aligned}
& \bar{\Omega}_{p}(h)=\left[\begin{array}{ccc}
{\left[\Omega_{p i j}(h)\right]_{7 \times 7}} & \epsilon_{1} \vartheta_{p} & v_{p}^{T} \\
* & -\epsilon_{1} I_{N} & 0 \\
* & * & -\epsilon_{1} I_{N}
\end{array}\right]<0, \\
& c_{1} e^{\delta T^{*}}\left(\lambda_{1}+\tau_{1} \lambda_{2}+\tau_{2} \lambda_{3}+\tau_{2} \lambda_{4}+\tau_{1}^{3} \lambda_{5}+\tau_{12}^{3} \lambda_{6}\right) \\
& -\lambda_{7} c_{2}<0
\end{aligned}
$$

where

$$
\begin{aligned}
& \Omega_{p 11}(h)=\left(I_{N} \otimes P_{p}\right)\left(I_{N} \otimes A\right) \\
& +\left(I_{N} \otimes A\right)^{T}\left(I_{N} \otimes P_{p}\right)^{T} \\
& +\left(I_{N} \otimes P_{p}\right)\left(I_{N} \otimes K_{p}\right) \\
& +\left(I_{N} \otimes K_{p}\right)^{T}\left(I_{N} \otimes P_{p}\right)^{T}+\left(I_{N} \otimes Q_{1}\right) \\
& +\left(I_{N} \otimes Q_{2}\right)+\left(I_{N} \otimes Q_{3}\right) \\
& +\tau_{1}^{2}\left(I_{N} \otimes R_{1}\right)+\kappa_{p}\left(I_{N} \otimes R_{3}\right) \\
& +\left(I_{N} \otimes \mathscr{G}\right)\left(I_{N} \otimes \mathscr{G}\right)^{T} \\
& +\sum_{q=1}^{\mathcal{N}} \pi_{p q}(h)\left(I_{N} \otimes P_{q}\right), \\
& \Omega_{p 13}=\left(I_{N} \otimes P_{p}\right) \lambda\left(T_{p} \otimes \Lambda\right), \\
& \Omega_{p 15}=\left(I_{N} \otimes P_{p}\right), \\
& \Omega_{p 22}=-\left(I_{N} \otimes Q_{1}\right)-\tau_{12}^{2}\left(I_{N} \otimes R_{2}\right), \\
& \Omega_{p 33}=-(1-\mu)\left(I_{N} \otimes Q_{2}\right)+\kappa_{p}\left(I_{N} \otimes R_{4}\right) \text {, } \\
& \Omega_{p 44}=-\left(I_{N} \otimes Q_{3}\right), \\
& \Omega_{p 55}=-\left(I_{N} \otimes I_{N}\right), \\
& \Omega_{p 66}=-\left(I_{N} \otimes R_{1}\right), \\
& \Omega_{p 77}=-\left(I_{N} \otimes R_{2}\right), \\
& \vartheta_{p}=[\bar{\alpha}\left(I_{N} \otimes P_{p}\right)\left(I_{N} \otimes M_{p}\right) \underbrace{\left.\begin{array}{llll}
0 & 0 & \cdots & 0
\end{array}\right]^{T},}_{6}
\end{aligned}
$$

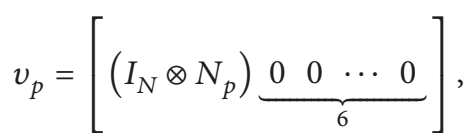

$$
\begin{aligned}
& \lambda_{1}=\max \left\{\lambda_{\max }\left(I_{N} \otimes \widetilde{P}_{p}\right), p \in \mathbb{S}\right\}, \\
& \lambda_{2}=\max \left\{\lambda_{\max }\left(I_{N} \otimes \widetilde{Q}_{1 p}\right), p \in \mathbb{S}\right\}, \\
& \lambda_{3}=\max \left\{\lambda_{\max }\left(I_{N} \otimes \widetilde{Q}_{2 p}\right), p \in \mathbb{S}\right\},
\end{aligned}
$$




$$
\begin{aligned}
& \lambda_{4}=\max \left\{\lambda_{\max }\left(I_{N} \otimes \widetilde{Q}_{3 p}\right), p \in \mathbb{S}\right\}, \\
& \lambda_{5}=\max \left\{\lambda_{\max }\left(I_{N} \otimes \widetilde{R}_{1 p}\right), p \in \mathbb{S}\right\}, \\
& \lambda_{6}=\max \left\{\lambda_{\max }\left(I_{N} \otimes \widetilde{R}_{2 p}\right), p \in \mathbb{S}\right\}, \\
& \lambda_{7}=\min \left\{\lambda_{\min }\left(I_{N} \otimes \widetilde{P}_{p}\right), p \in \mathbb{S}\right\}, \\
& \tau_{12}=\tau_{2}-\tau_{1},
\end{aligned}
$$

and the rest of elements of $\Omega_{p i j}(h)$ are zero.

Proof. To develop the finite-time stochastic synchronization criterion for the network model (1), it is enough to establish the finite-time stochastic stability criterion for the closedloop error system (5). For this purpose, we select the Lyapunov-Krasovskii functional as follows:

$$
V\left(e_{t}, t, p\right)=\sum_{l=1}^{3} V_{l}\left(e_{t}, t, p\right),
$$

where

$$
\begin{aligned}
& V_{1}\left(e_{t}, t, p\right)=e^{T}(t)\left(I_{N} \otimes P_{p}\right) e(t), \\
& V_{2}\left(e_{t}, t, p\right) \\
& \quad=\int_{t-\tau_{1}}^{t} e^{T}(s)\left(I_{N} \otimes Q_{1}\right) e(s) d s \\
& \quad+\int_{t-\tau(t)}^{t} e(s)\left(I_{N} \otimes Q_{2}\right) e(s) d s \\
& \quad+\int_{t-\tau_{2}}^{t} e^{T}(s)\left(I_{N} \otimes Q_{3}\right) e(s) d s, \\
& V_{3}\left(e_{t}, t, p\right) \\
& =\tau_{1} \int_{-\tau_{1}}^{0} \int_{t+\theta}^{t} e^{T}(s)\left(I_{N} \otimes R_{1}\right) e(s) d s d \theta \\
& \quad+\tau_{12} \int_{-\tau_{2}}^{-\tau_{1}} \int_{t+\theta}^{t-\tau_{1}} e^{T}(s)\left(I_{N} \otimes R_{2}\right) e(s) d s d \theta,
\end{aligned}
$$

with $P_{p}>0, Q_{l}(l=1,2,3), R_{v}(v=1,2)$ and $\tau_{12}=\tau_{2}-\tau_{1}$.

Based on Ito's differential formula [9], the stochastic derivative of $V\left(e_{t}, t, p\right)$ can be obtained as

$$
\begin{aligned}
d V\left(e_{t}, t, p\right) & \\
= & \mathfrak{E} V\left(e_{t}, t, p\right) \\
& +V_{e}\left(e_{t}, t, p\right) \rho(t, e(t), e(t-\tau(t))) d w(t),
\end{aligned}
$$

where $£ V\left(e_{t}, t, p\right)=£ V_{1}\left(e_{t}, t, p\right)+£ V_{2}\left(e_{t}, t, p\right)+£ V_{3}\left(e_{t}, t, p\right)$ and $V_{e}\left(e_{t}, t, p\right)=\partial V\left(e_{t}, t, p\right) / \partial e$.
Now, by calculating the time derivative of $V\left(e_{t}, t, p\right)$ along the solution trajectories of the error system (5), we can get

$$
\begin{gathered}
£ V_{1}\left(e_{t}, t, p\right)=2 e^{T}(t)\left(I_{N} \otimes P_{p}\right)\left[\left(I_{N} \otimes A\right)+\left(I_{N}\right.\right. \\
\left.\otimes K_{p}\right)+\alpha(t)\left(I_{N} \otimes M_{p} \Gamma_{p}(t) N_{p}\right)+(\alpha(t)-\bar{\alpha}) \\
\times\left(I_{N} \otimes M_{p} \Gamma_{p}(t) N_{p}\right) e(t)+\lambda\left(T_{p} \otimes \Lambda\right) e(t \\
-\tau(t))+G(t, e(t))]+\sum_{q=1}^{\mathcal{N}} \pi_{p q}(h) e^{T}(t)\left(I_{N} \otimes P_{q}\right) \\
\cdot e(t)+\operatorname{trace}\left\{\rho^{T}(t, e(t), e(t-\tau(t)))\left(I_{N} \otimes P_{p}\right)\right. \\
\cdot \rho(t, e(t), e(t-\tau(t)))\}, \\
£ V_{2}\left(e_{t}, t, p\right) \leq e^{T}(t)\left(\left(I_{N} \otimes Q_{1}\right)+\left(I_{N} \otimes Q_{2}\right)+\left(I_{N}\right.\right. \\
\left.\left.\otimes Q_{3}\right)\right) e(t)-e^{T}\left(t-\tau_{1}\right)\left(I_{N} \otimes Q_{1}\right) e\left(t-\tau_{1}\right)-(1 \\
-\mu) e^{T}(t-\tau(t))\left(I_{N} \otimes Q_{2}\right) e(t-\tau(t))-e^{T}(t \\
\left.-\tau_{2}\right)\left(I_{N} \otimes Q_{3}\right) e\left(t-\tau_{2}\right), \\
£ V_{3}\left(e_{t}, t, p\right)=\tau_{1}^{2} e^{T}(t)\left(I_{N} \otimes R_{1}\right) e(t)+\tau_{12}^{2} e^{T}\left(t-\tau_{1}\right) \\
\cdot\left(I_{N} \otimes R_{2}\right) e\left(t-\tau_{1}\right)-\tau_{1} \int_{t-\tau_{1}}^{t} e^{T}(s)\left(I_{N} \otimes R_{1}\right) \\
\cdot e(s) d s-\tau_{12} \int_{t-\tau_{2}}^{t-\tau_{1}} e^{T}(s)\left(I_{N} \otimes R_{2}\right) e(s) d s .
\end{gathered}
$$

Further, by applying Jensen's single integral inequality [6] to the integral terms in (16), we can get the following inequalities:

$$
\begin{aligned}
& -\tau_{1} \int_{t-\tau_{1}}^{t} e^{T}(s)\left(I_{N} \otimes R_{1}\right) e(s) d s \\
& \leq-\int_{t-\tau_{1}}^{t} e^{T}(s) d s\left(I_{N} \otimes R_{1}\right) \int_{t-\tau_{1}}^{t} e(s) d s, \\
& -\tau_{12} \int_{t-\tau_{2}}^{t-\tau_{1}} e^{T}(s)\left(I_{N} \otimes R_{2}\right) e(s) d s \\
& \quad \leq-\int_{t-\tau_{2}}^{t-\tau_{1}} e^{T}(s) d s\left(I_{N} \otimes R_{2}\right) \int_{t-\tau_{2}}^{t-\tau_{1}} e(s) d s .
\end{aligned}
$$

On the other hand, it follows from Assumption 2 and condition (7) that

$$
\begin{aligned}
& \operatorname{trace}\left\{\rho^{T}(t, e(t), e(t-\tau(t)))\left(I_{N} \otimes P_{p}\right)\right. \\
& \cdot \rho(t, e(t), e(t-\tau(t)))\} \leq \kappa_{p} \\
& \cdot \operatorname{trace}\left\{\rho^{T}(t, e(t), e(t-\tau(t)))\right. \\
& \cdot \rho(t, e(t), e(t-\tau(t)))\} \leq \kappa_{p}\left(e^{T}(t)\left(I_{N} \otimes R_{3}\right)\right. \\
& \left.\cdot e(t)+e^{T}(t-\tau(t))\left(I_{N} \otimes R_{4}\right) e(t-\tau(t))\right),
\end{aligned}
$$


where $\kappa_{p}(p \in \mathbb{S})$ are positive scalars and $R_{3}, R_{4}$ are known constant matrices.

Moreover, according to Assumption 1, we can obtain the following inequality:

$$
\begin{aligned}
e^{T}(t) & \left(I_{N} \otimes \mathscr{G}\right)\left(I_{N} \otimes \mathscr{G}\right)^{T} e(t) \\
& -\mathbb{F}^{T}(t, e(t)) \mathbb{F}(t, e(t))>0 .
\end{aligned}
$$

Then, by combining (13)-(19) and taking mathematical expectation, it can be obtained that

$$
\begin{aligned}
& \frac{\mathbb{E}\left\{d V\left(e_{t}, t, p\right)\right\}}{d t} \leq \mathbb{E}\left\{£ V\left(e_{t}, p, t\right)\right\} \leq \mathbb{E}\left\{\xi^{T}(t)\right. \\
& \cdot\left(\left[\Omega_{p i j}(h)\right]_{7 \times 7}+\vartheta_{p} \Gamma_{p}(t) v_{p}+\left(\vartheta_{p} \Gamma_{p}(t) v_{p}\right)^{T}\right) \\
& \cdot \xi(t)\},
\end{aligned}
$$

where

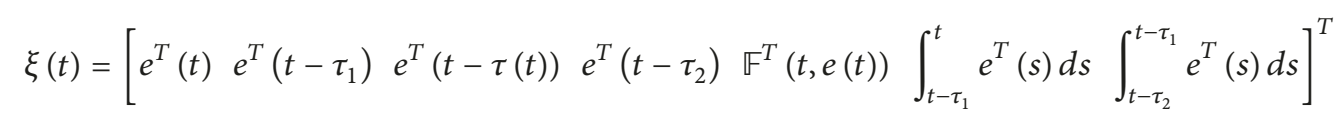

and the elements of $\Omega_{p i j}(h), \vartheta_{p}$, and $v_{p}$ are defined in the theorem statement. Moreover, based on Lemma 2 in [6], for any positive scalar $\epsilon_{1}$, the right-hand side of (20) can equivalently be written as

$$
\begin{gathered}
{\left[\Omega_{p i j}(h)\right]_{7 \times 7}+\vartheta_{p} \Gamma_{p}(t) v_{p}+\left(\vartheta_{p} \Gamma_{p}(t) v_{p}\right)^{T}} \\
\leq\left[\Omega_{p i j}(h)\right]_{7 \times 7}+\epsilon_{1} \vartheta_{p} \vartheta_{p}^{T}+\epsilon_{1}^{-1} v_{p}^{T} v_{p} .
\end{gathered}
$$

Based on the Schur complement, it is noted that (22) is equivalent to the left-hand side of (8). Thus, it can be observed

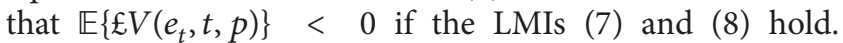
Furthermore, if there exists a constant $\delta>0$, it yields that $\mathbb{E}\left\{\mathfrak{E}\left(e_{t}, t, p\right)\right\}<\delta \mathbb{E}\left\{V\left(e_{t}, t, p\right)\right\}$. From which, it can be obtained that $\mathbb{E}\left\{e^{-\delta t} V\left(e_{t}, t, p\right)\right\}<\mathbb{E}\left\{V\left(e_{0}, 0, p_{0}\right)\right\}$, where $p_{0}=\sigma(0)$. Next, define the following new parameters: $\widetilde{P}_{p}=$ $Z_{p}^{-1 / 2} P_{p} Z_{p}^{-1 / 2}, \widetilde{Q}_{1 p}=Z_{p}^{-1 / 2} Q_{1} Z_{p}^{-1 / 2}, \widetilde{Q}_{2 p}=Z_{p}^{-1 / 2} Q_{2} Z_{p}^{-1 / 2}$, $\widetilde{Q}_{3 p}=Z_{p}^{-1 / 2} Q_{3} Z_{p}^{-1 / 2}, \widetilde{R}_{1 p}=Z_{p}^{-1 / 2} R_{1} Z_{p}^{-1 / 2}, \widetilde{R}_{2 p}=$ $Z_{p}^{-1 / 2} R_{2} Z_{p}^{-1 / 2}$. Then, it follows from condition (11) and $0 \leq$ $t \leq T^{*}$ that

$$
\begin{aligned}
& \mathbb{E}\left\{V\left(e_{t}, t, p\right)\right\}<e^{\delta t}\left\{V\left(e_{0}, 0, p_{0}\right)\right\} \\
& \leq e^{\delta t}\left\{e^{T}(0)\left(I_{N} \otimes P_{p}\right) e(0)\right. \\
& +\int_{-\tau_{1}}^{0} e^{T}(s)\left(I_{N} \otimes Q_{1}\right) e(s) d s \\
& +\int_{-\tau(0)}^{0} e^{T}(s)\left(I_{N} \otimes Q_{2}\right) e(s) d s \\
& +\int_{-\tau_{2}}^{0} e^{T}(s)\left(I_{N} \otimes Q_{3}\right) e(s) d s \\
& +\tau_{1}^{2} \int_{-\tau_{1}}^{0} e^{T}(s)\left(I_{N} \otimes R_{1}\right) e(s) d s \\
& \left.+\tau_{12}^{2} \int_{-\tau_{2}}^{0} e^{T}(s)\left(I_{N} \otimes R_{2}\right) e(s) d s\right\}
\end{aligned}
$$

$$
\begin{aligned}
& =e^{\delta T^{*}}\left(\max \left\{\lambda_{\max }\left(I_{N} \otimes \widetilde{P}_{p}\right)\right\}\right. \\
& +\tau_{1} \max \left\{\lambda_{\max }\left(I_{N} \otimes \widetilde{Q}_{1 p}\right)\right\} \\
& +\tau_{2} \max \left\{\lambda_{\max }\left(I_{N} \otimes \widetilde{Q}_{2 p}\right)\right\} \\
& +\tau_{2} \max \left\{\lambda_{\max }\left(I_{N} \otimes \widetilde{Q}_{3 p}\right)\right\} \\
& +\tau_{1}^{3} \max \left\{\lambda_{\max }\left(I_{N} \otimes \widetilde{R}_{1 p}\right)\right\} \\
& \left.+\tau_{12}^{3} \max \left\{\lambda_{\max }\left(I_{N} \otimes \widetilde{R}_{2 p}\right)\right\}\right) \\
& \times \mathbb{E}\left\{e^{T}(s)\left(I_{N} \otimes Z_{p}\right) e(s)\right\} \leq c_{1} e^{\delta T^{*}}\left(\lambda_{1}+\tau_{1} \lambda_{2}\right. \\
& \left.+\tau_{2} \lambda_{3}+\tau_{2} \lambda_{4}+\tau_{1}^{3} \lambda_{5}+\tau_{12}^{3} \lambda_{6}\right)
\end{aligned}
$$

where $\lambda_{a}(a=1,2, \ldots, 6)$ are defined in the theorem statement and $c_{1}$ is given in Definition 4 .

On the other hand, from (11), we can have

$$
\begin{aligned}
& \mathbb{E}\left\{V\left(e_{t}, t, p\right)\right\} \geq \mathbb{E}\left\{e^{T}(t)\left(I_{N} \otimes P_{p}\right) e(t)\right\} \\
& \quad=\min \left\{\lambda_{\min }\left(I_{N} \otimes \widetilde{P}_{p}\right)\right\} \mathbb{E}\left\{e^{T}(t)\left(I_{N} \otimes Z_{p}\right) e(t)\right\} \\
& \quad \geq \lambda_{7} \mathbb{E}\left\{e^{T}(t)\left(I_{N} \otimes Z_{p}\right) e(t)\right\} .
\end{aligned}
$$

Now, by combining the inequalities (23) and (24), we can get

$$
\begin{aligned}
& \mathbb{E}\left\{e^{T}(t)\left(I_{N} \otimes Z_{p}\right) e(t)\right\} \\
& \quad \leq \frac{c_{1}\left(\lambda_{1}+\tau_{1} \lambda_{2}+\tau_{2} \lambda_{3}+\tau_{2} \lambda_{4}+\tau_{1}^{3} \lambda_{5}+\tau_{12}^{3} \lambda_{6}\right)}{e^{-\delta T^{*}} \lambda_{7}} \\
& \quad<c_{2} .
\end{aligned}
$$

It is clear to see that the inequality (25) is the same as that in (9) which is the desired condition. Hence, it can be concluded that the closed-loop error system (5) is finitetime stochastically stable which means that the considered network model (1) is stochastically synchronized within 
a prescribed finite-time interval. Thus, the proof of this theorem is completed.

Theorem 6. Consider the network model (1) with Assumptions $1-3$. For given positive scalars $c_{1}, c_{2}, T^{*}, \delta, \mu, \lambda, \bar{\alpha} \in[0,1], \tau_{1}, \tau_{2}$, symmetric matrix $Z_{p}(p \in \mathbb{S})$ and diagonal matrices $R_{3}, R_{4}$, the considered network (1) is stochastically synchronized in finitetime under the non-fragile state feedback controller (3), if there exist symmetric matrices $X_{p}>0(p \in \mathbb{S}), \widehat{Q}_{l p}>0(l=1,2,3)$, $\widehat{R}_{v p}>0(v=1,2)$, any matrices $Y_{p}(p \in \mathbb{S})$ with appropriate dimensions and positive scalars $\widehat{\kappa}_{p},(p \in \mathbb{S}), r, a_{l}(l=1,2,3)$, $b_{v}(v=1,2), \epsilon_{1}$ such that the following matrix inequalities hold:

$$
\begin{aligned}
& X_{p}-\widehat{\kappa}_{p} I_{N}>0, \quad p \in \mathbb{S}, \\
& \widehat{\bar{\Omega}}_{p}(h)=\left[\widehat{\Omega}_{p i j}(h)\right]_{12 \times 12}<0, \\
& r Z_{p}^{-1}<X_{p}<Z_{p}^{-1}, \\
& 0<\widehat{Q}_{l p}<a_{l} Z_{p}^{-1}, \\
& 0<\widehat{R}_{v p}<b_{v} Z_{p}^{-1}, \\
& {\left[\begin{array}{ccc}
c_{1}\left(\tau_{1} a_{1}+\tau_{2} a_{2}+\tau_{2} a_{3}+\tau_{1}^{3} b_{1}+\tau_{12}^{3} b_{2}\right)-e^{\delta T^{*}} c_{2} & \sqrt{c_{1}} \\
* & -r
\end{array}\right]}
\end{aligned}
$$

$<0$,

where

$$
\begin{aligned}
& \widehat{\Omega}_{p 11}(h)=\left(I_{N} \otimes A\right)\left(I_{N} \otimes X_{p}\right)+\left(I_{N} \otimes X_{p}\right)\left(I_{N}\right. \\
& \otimes A)^{T}+\left(I_{N} \otimes Y_{p}\right)+\left(I_{N} \otimes Y_{p}\right)^{T}+\left(I_{N} \otimes \widehat{Q}_{1 p}\right) \\
& +\left(I_{N} \otimes \widehat{Q}_{2 p}\right)+\left(I_{N} \otimes \widehat{Q}_{3 p}\right)+\tau_{1}^{2}\left(I_{N} \otimes \widehat{R}_{1 p}\right) \\
& +\pi_{p p}(h)\left(I_{N} \otimes X_{p}\right), \\
& \widehat{\Omega}_{p 13}=\lambda\left(T_{p} \otimes \Lambda\right)\left(I_{N} \otimes X_{p}\right), \\
& \widehat{\Omega}_{p 15}=\left(I_{N} \otimes I_{N}\right), \\
& \widehat{\Omega}_{p 18}=\bar{\alpha}\left(I_{N} \otimes M_{p}\right), \\
& \widehat{\Omega}_{p 19}=\left(I_{N} \otimes X_{p}\right)\left(I_{N} \otimes N_{p}\right)^{T}, \\
& \widehat{\Omega}_{p 110}=\left(I_{N} \otimes X_{p}\right) \sqrt{\left(I_{N} \otimes R_{3}\right)}, \\
& \widehat{\Omega}_{p 111}=\left(I_{N} \otimes X_{p}\right)\left(I_{N} \otimes \mathscr{G}\right), \\
& \widehat{\Omega}_{p 112}=\left[\sqrt{\pi_{p 1(h)}} X_{p}^{T}, \ldots, \sqrt{\pi_{p(p-1)(h)}} X_{p}^{T},\right. \\
& \left.\sqrt{\pi_{p(p+1)(h)}} X_{p}^{T}, \ldots, \sqrt{\pi_{p \mathcal{N}(h)}} X_{p}^{T}\right], \\
& \widehat{\Omega}_{p 22}=-\left(I_{N} \otimes \widehat{Q}_{1 p}\right)-\tau_{12}^{2}\left(I_{N} \otimes \widehat{R}_{2 p}\right) \text {, }
\end{aligned}
$$

$$
\begin{aligned}
& \widehat{\Omega}_{p 33}=-(1-\mu)\left(I_{N} \otimes \widehat{Q}_{2 p}\right), \\
& \widehat{\Omega}_{p 310}=\left(I_{N} \otimes X_{p}\right) \sqrt{\left(I_{N} \otimes R_{4}\right)}, \\
& \widehat{\Omega}_{p 44}=-\left(I_{N} \otimes \widehat{Q}_{3 p}\right), \\
& \widehat{\Omega}_{p 55}=-\left(I_{N} \otimes I_{N}\right), \\
& \widehat{\Omega}_{p 66}=-\left(I_{N} \otimes \widehat{R}_{1 p}\right), \\
& \widehat{\Omega}_{p 77}=-\left(I_{N} \otimes \widehat{R}_{2 p}\right), \\
& \widehat{\Omega}_{p 88}=-\epsilon_{1}\left(I_{N} \otimes I_{N}\right), \\
& \widehat{\Omega}_{p 99}=-\epsilon_{1}\left(I_{N} \otimes I_{N}\right), \\
& \widehat{\Omega}_{p 1010}=-\widehat{\kappa}_{p}\left(I_{N} \otimes I_{N}\right), \\
& \widehat{\Omega}_{p 1111}=-\left(I_{N} \otimes I_{N}\right), \\
& \widehat{\Omega}_{p 1212}=-\operatorname{diag}\left\{X_{1}, \ldots, X_{p-1}, X_{p+1}, \ldots, X_{\mathcal{N}}\right\}, \\
& \widehat{\kappa}_{p}=\frac{-1}{\kappa_{p}},
\end{aligned}
$$

and the remaining parameters of $\widehat{\Omega}_{p i j}(h)$ are zero. Moreover, if the obtained LMIs are feasible, then the desired state feedback controller gain matrices in (3) are computed by $K_{p}=Y_{p} X_{p}^{-1}$.

Proof. Let $X_{p}=P_{p}^{-1}$ and pre- and postmultiply the matrix $\bar{\Omega}_{p}(h)$ by $\operatorname{diag}\{\underbrace{}_{\left(I_{N} \otimes X_{p}\right), \ldots,\left(I_{N} \otimes X_{p}\right.}),\left(I_{N} \otimes I_{N}\right),\left(I_{N} \otimes\right.$ $\left.\left.X_{p}\right),\left(I_{N} \otimes X_{p}\right),\left(I_{N} \otimes I_{N}\right),\left(I_{N} \otimes I_{N}\right)\right\}$. Now, we introduce the following new variables: $X_{p} Q_{l} X_{p}=\widehat{Q}_{l p}(l=1,2,3)$, $X_{p} R_{v} X_{p}=\widehat{R}_{v p}(v=1,2)$, and $Y_{p}=K_{p} X_{p}$.

Moreover, from Theorem 5, it is noticed that $\lambda_{7}<\left(I_{N} \otimes\right.$ $\left.Z_{p}^{-1 / 2} P_{p} Z_{p}^{-1 / 2}\right)<\lambda_{1}, 0<\left(I_{N} \otimes Z_{p}^{-1 / 2} Q_{1} Z_{p}^{-1 / 2}\right)<\lambda_{2}, 0<$ $\left(I_{N} \otimes Z_{p}^{-1 / 2} Q_{2} Z_{p}^{-1 / 2}\right)<\lambda_{3}, 0<\left(I_{N} \otimes Z_{p}^{-1 / 2} Q_{3} Z_{p}^{-1 / 2}\right)<\lambda_{4}, 0<$ $\left(I_{N} \otimes Z_{p}^{-1 / 2} R_{1} Z_{p}^{-1 / 2}\right)<\lambda_{5}$ and $0<\left(I_{N} \otimes Z_{p}^{-1 / 2} R_{2} Z_{p}^{-1 / 2}\right)<\lambda_{6}$. According to the congruence transformation, these relations can be changed into $\lambda_{1}^{-1}\left(I_{N} \otimes Z_{p}^{-1}\right)<\left(I_{N} \otimes X_{p}\right)<\lambda_{7}^{-1}\left(I_{N} \otimes\right.$ $\left.Z_{p}^{-1}\right), 0<\left(I_{N} \otimes \widehat{Q}_{l p}\right)<\lambda_{7}^{-2} \lambda_{l+1}\left(I_{N} \otimes Z_{p}^{-1}\right)(l=1,2,3)$ and $0<\left(I_{N} \otimes \widehat{R}_{v p}\right)<\lambda_{7}^{-2} \lambda_{v+4}\left(I_{N} \otimes Z_{p}^{-1}\right)(v=1,2)$. Now, if we set $\lambda_{7}=1, \lambda_{1}^{-1}=r, \lambda_{l+1} \leq a_{l}(l=1,2,3)$ and $\lambda_{v+4} \leq b_{v}(v=1,2)$, then the constraints in (28) can easily be deduced. Moreover, the conditions in (26), (27), and (29) can be obtained from (7), (8), and (9), respectively, which are the desired conditions. Hence, the proof is completed.

Remark 7. It should be mentioned that the constraints in (27) are cannot be solved directly via MATLAB LMI control toolbox due to the existence of the time-varying terms $\sum_{q=1}^{\mathcal{N}} \pi_{p q}(h)$. To overcome this difficulty, the transition rates $\pi_{p q}(h)$ are assumed to be bounded and satisfy 
$\pi_{p q}^{-} \leq \pi_{p q}(h) \leq \pi_{p q}^{+}$, since they are partially measurable in practice which is mentioned in [26]. Moreover, in this case, the following assumptions are made as in [26]:

$$
\begin{aligned}
\pi_{p q}(h)= & \sum_{k=1}^{\mathcal{N}} \gamma_{k} \pi_{p q, k}, \quad \sum_{k=1}^{\mathcal{N}} \gamma_{k}=1, \gamma_{k} \geq 0, \\
\pi_{p q, k} & = \begin{cases}\pi_{p q}^{-}+(k-1) \frac{\pi_{p q}^{+}-\pi_{p q}^{-}}{\mathcal{N}-1}, & p \neq q, q \in \mathbb{S}, \\
\pi_{p q}^{+}-(k-1) \frac{\pi_{p q}^{+}-\pi_{p q}^{-}}{\mathcal{N}-1}, & p=q, q \in \mathbb{S} .\end{cases}
\end{aligned}
$$

Now, we are able to present the sufficient conditions guaranteeing the stochastic synchronization of the considered network (1) over a finite-time interval in terms of LMIs in which all the elements are either constants or constant matrices according to Remark 7. Thus, we have the following theorem.

Theorem 8. For given positive scalars $c_{1}, c_{2}, T^{*}, \delta, \lambda, \mu, \bar{\alpha} \in$ $[0,1], \tau_{1}, \tau_{2}$, symmetric matrix $Z_{p}(p \in \mathbb{S})$ and diagonal matrices $R_{3}, R_{4}$, the considered network (1) with Assumptions 1-3 is stochastically synchronized in finite-time under the nonfragile controller (3), if there exist symmetric matrices $X_{p}>$ $0(p \in \mathbb{S}), \widehat{Q}_{l p}>0(l=1,2,3), \widehat{R}_{v p}>0(v=1,2)$, any matrices $Y_{p}(p \in \mathbb{S})$ with appropriate dimensions and positive scalars $\widehat{\kappa}_{p}(p \in \mathbb{S}), r, a_{l}(l=1,2,3), b_{v}(v=1,2), \epsilon_{1}$ such that the following matrix inequality, (26), (28), and (29) hold:

$$
\left[\widehat{\Omega}_{p i j, k}\right]_{12 \times 12}<0, \quad k=1,2, \ldots, \mathcal{N}
$$

where

$$
\begin{aligned}
& \widehat{\Omega}_{p 11, k}=\left(I_{N} \otimes A\right)\left(I_{N} \otimes X_{p}\right)+\left(I_{N} \otimes X_{p}\right)\left(I_{N} \otimes A\right)^{T} \\
& +\left(I_{N} \otimes Y_{p}\right)+\left(I_{N} \otimes Y_{p}\right)^{T}+\left(I_{N} \otimes \widehat{Q}_{1 p}\right)+\left(I_{N}\right. \\
& \left.\otimes \widehat{Q}_{2 p}\right)+\left(I_{N} \otimes \widehat{Q}_{3 p}\right)+\tau_{1}^{2}\left(I_{N} \otimes \widehat{R}_{1 p}\right)+\pi_{p p, k}\left(I_{N}\right. \\
& \left.\otimes X_{p}\right), \\
& \widehat{\Omega}_{p 112, k}=\left[\sqrt{\pi_{p 1, k}} X_{p}^{T}, \ldots, \sqrt{\pi_{p(p-1), k}} X_{p}^{T}, \sqrt{\pi_{p(p+1), k}} X_{p}^{T},\right. \\
& \left.\ldots, \sqrt{\pi_{p, N, k}} X_{p}^{T}\right],
\end{aligned}
$$

and the remaining elements of $\widehat{\Omega}_{p i j, k}$ are the same as those defined in Theorem 6. Further, the nonfragile state feedback controller gain matrices in (3) are calculated by $K_{p}=Y_{p} X_{p}^{-1}$.

Proof. Based on Remark 7, the time-varying element $\pi_{p q}(h)$ may take values in the interval $\left[\pi_{p q}^{-}, \pi_{p q}^{+}\right]$. Then, by using (31) and following the similar lines in the proof of Theorem 6 , it is easy to obtain the inequality (32) which completes the proof.

Remark 9. It should be noted that, so far in the literature, several control approaches have been proposed for the synchronization problem of several CDNs [3-6], wherein the interconnection topology among the nodes are assumed to be fixed. However, in practice, this assumption is practically difficult or even impossible. However, yet now, there were no results reported in the existing literature for the synchronization analysis of stochastic CDNs with switching topology. According to this fact, in this paper, finite-time synchronization problem of stochastic CDNs with switching topology is investigated. Furthermore, due to random behavior in the dynamics of stochastic CDNs, it is very difficult to determine the exact fixed control value. Therefore, in this paper, the feedback control gain is considered with uncertain terms, which is more significant to reflect the realistic scenarios.

\section{An Illustrative Example}

This section provides an illustrative example to verify the developed theoretical results in the previous section. For the sake of simplicity, consider a class of CDNs in the form of (1) with five identical nodes and the state vector of each node being three-dimensional, that is, $N=5$ and $n=3$.

Let us select the network matrix and the nonlinear function as

$$
\begin{aligned}
A & =\left[\begin{array}{ccc}
1 & -1.4 & 0 \\
-0.92 & 1.2 & -0.5 \\
0 & 2 & -1.2
\end{array}\right], \\
f\left(t, x_{i}(t)\right) & =\left[\begin{array}{l}
0.5 \exp (-0.01 t) \tanh \left(x_{i 1}(t)\right) \\
0.5 \exp (-0.01 t) \tanh \left(x_{i 2}(t)\right) \\
0.5 \exp (-0.01 t) \tanh \left(x_{i 3}(t)\right)
\end{array}\right] .
\end{aligned}
$$

It is clear to see that $f\left(t, x_{i}(t)\right)$ satisfies Assumption 1 with $\mathscr{G}=\operatorname{diag}\{0.5,0.5,0.5\}$. In this example, we consider the semiMarkov jump topology with two modes, whose connectivity graph is shown in Figure 1. The inner coupling matrix is assumed to be $\Lambda=\operatorname{diag}\{0.2,0.5,0.7\}$ and the coupling strength is chosen as $\lambda=0.5$. The time-varying delay is taken as $\tau(t)=0.5+0.5 \sin (t)$ from which it can be obtained that $\tau_{1}=0, \tau_{2}=1$ and $\mu=0.5$.

Based on Figure 1, the jumping coupling configuration matrices $T(\sigma(t))$ for $\sigma(t)=1,2$ can be expressed as

$$
\begin{aligned}
& T_{1}=\left[\begin{array}{ccccc}
0 & 0 & 0 & 0 & 0 \\
1 & -1 & 0 & 0 & 0 \\
0 & 1 & -1 & 0 & 0 \\
1 & 0 & 0 & -1 & 0 \\
0 & 0 & 0 & 1 & -1
\end{array}\right], \\
& T_{2}=\left[\begin{array}{ccccc}
-1 & 1 & 0 & 0 & 0 \\
0 & 0 & 0 & 0 & 0 \\
1 & 0 & -1 & 0 & 0 \\
0 & 0 & 1 & -1 & 0 \\
0 & 0 & 1 & 0 & 1
\end{array}\right] .
\end{aligned}
$$

Moreover, the elements $\pi_{12}(h)$ and $\pi_{21}(h)$ of transition rate matrix are assumed to lie in the intervals $[0.1,2]$ and $[0.8,1.7]$, 


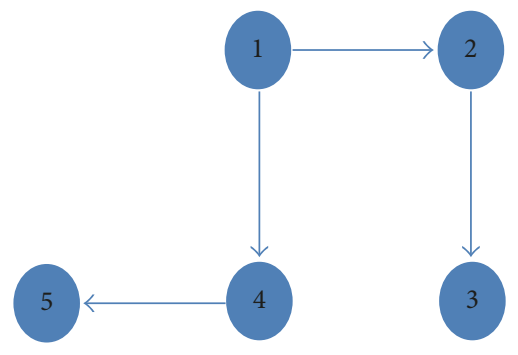

(a)

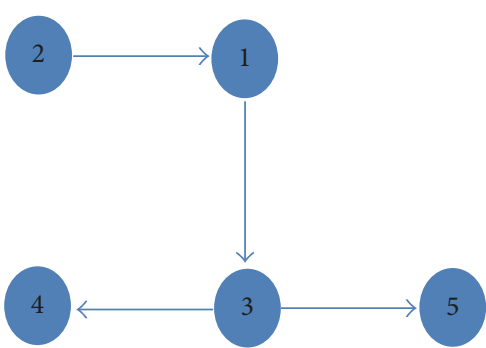

(b)

FIgURE 1: Connected topology of the considered CDNs.

respectively. So, in light of (31), the transition rates $\pi_{12}(h)$ and $\pi_{21}(h)$ can be represented as $\pi_{12}(h)=\sum_{n=1}^{2} \gamma_{n} \pi_{12, k}$ and $\pi_{21}(h)=\sum_{n=1}^{2} \gamma_{n} \pi_{21, k}$, respectively, with $\pi_{12,1}=0.1, \pi_{12,2}=$ 2 , $\pi_{21,1}=0.8$, and $\pi_{21,2}=1.7$. The stochastic variable representing the controller gain fluctuations is chosen as $\alpha(t)=0.25+0.25 \sin (t)$. Furthermore, the uncertain parameter matrices in the control gain are taken as

$$
\begin{aligned}
& M_{1}=\left[\begin{array}{lll}
0.01 & 0.01 & 0.02 \\
0.02 & 0.02 & 0.01 \\
0.02 & 0.01 & 0.10
\end{array}\right], \\
& M_{2}=\left[\begin{array}{lll}
0.01 & 0.02 & 0.02 \\
0.02 & 0.03 & 0.01 \\
0.02 & 0.01 & 0.01
\end{array}\right], \\
& N_{1}=\left[\begin{array}{lll}
0.01 & 0.02 & 0.03 \\
0.01 & 0.03 & 0.01 \\
0.02 & 0.02 & 0.01
\end{array}\right], \\
& N_{2}=\left[\begin{array}{lll}
0.02 & 0.01 & 0.02 \\
0.01 & 0.01 & 0.01 \\
0.02 & 0.01 & 0.02
\end{array}\right]
\end{aligned}
$$

and $\Gamma_{p}(t)=\sin (t)$. The rest of parameters involved in the simulation are set to be $c_{1}=1, c_{2}=2, \delta=0.01$, and $T^{*}=1$. Then, by solving the LMIs (26), (28), and (29) in Theorem 6 along with (32) in Theorem 8 with the aid of MATLAB LMI control toolbox, we can get a set of feasible solutions from which the nonfragile state feedback control gain matrices can be obtained as follows:

$$
\begin{aligned}
& K_{1}=\left[\begin{array}{ccc}
-17.7856 & 172.5392 & -19.7897 \\
-155.4332 & -30.3290 & 66.8671 \\
17.6228 & -66.6954 & -40.4541
\end{array}\right], \\
& K_{2}=\left[\begin{array}{ccc}
-19.2269 & -2.5815 & -51.2629 \\
4.3104 & -29.1095 & -64.4932 \\
39.9849 & 57.5098 & -35.7536
\end{array}\right] .
\end{aligned}
$$

Here, our aim is to design the nonfragile state feedback controller such that the considered network (1) is robustly

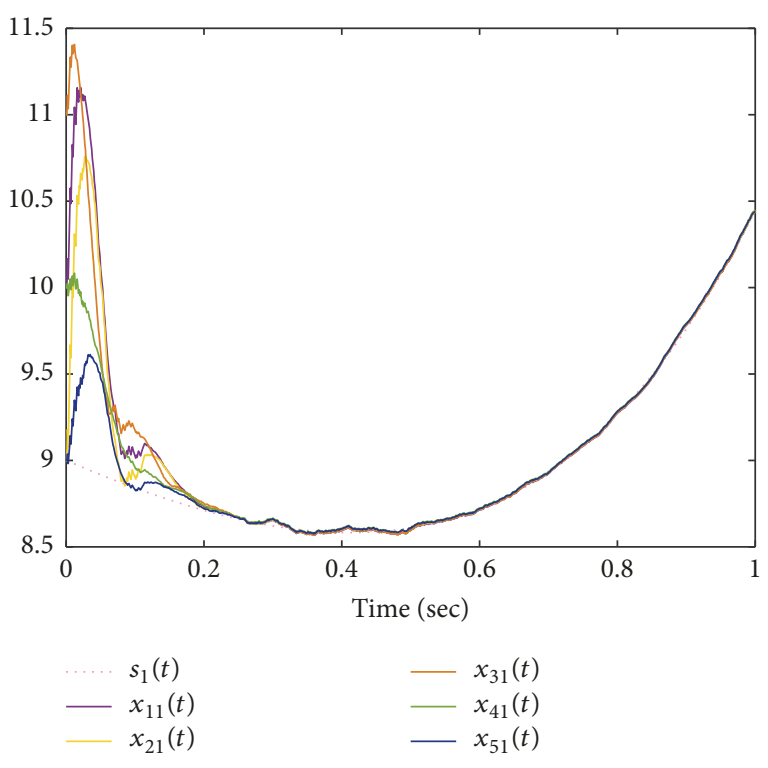

Figure 2: State responses of first node.

synchronized with the target network within a desired finitetime interval. For the simulation purposes, we set the initial conditions for the states of the nodes and the isolated node as follows: $x_{1}(0)=\left[\begin{array}{lll}10 & 11 & 10\end{array}\right]^{T}, x_{2}(0)=\left[\begin{array}{lll}9 & 11 & 10\end{array}\right]^{T}, x_{3}(0)=$ $\left[\begin{array}{lll}11 & 10 & 9\end{array}\right]^{T}, x_{4}(0)=\left[\begin{array}{lll}10 & 9 & 10\end{array}\right]^{T}, x_{5}(0)=\left[\begin{array}{lll}10 & 11 & 10\end{array}\right]^{T}$, and $s(0)=\left[\begin{array}{lll}9 & 8 & 9\end{array}\right]^{T}$. The noise intensity function is taken as $\rho\left(t, x_{i}(t), x_{i}(t-\tau(t))\right)=\left[0.1 \sin \left(x_{i 1}(t)\right) \quad 0.1 \sin \left(x_{i 2}(t-\right.\right.$ $\left.\tau(t))) 0.1 \sin \left(x_{i 3}(t-\tau(t))\right)\right]^{T}$.

Based on these values, simulations are drawn in Figures 2-12. Specifically, the state responses of the first, second, and third nodes together with the isolated node are plotted in Figures 2, 3, and 4, respectively, wherein the dotted line represents the isolated node and the dashed lines denote the five identical nodes. It can easily be observed from these figures that the states of the nodes are exactly synchronized with the states of the isolated node within short period which shows the efficiency of the proposed nonfragile control strategy. Moreover, the corresponding error state responses and the control response curves are given in Figures 5-7 and Figures 8-10, respectively. Further, Figure 11 shows the jumping mode of the semi-Markov switching topology. In 


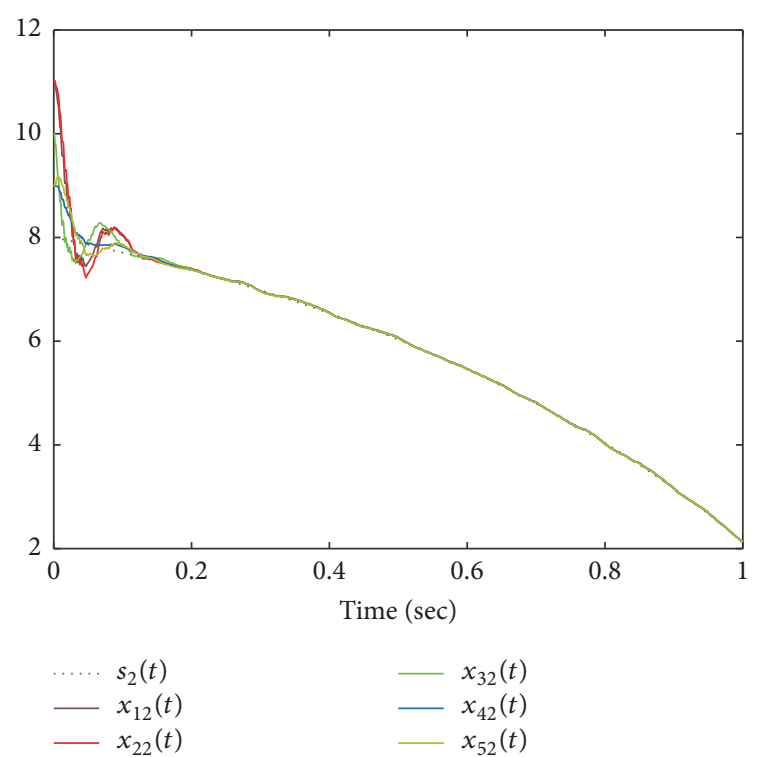

FIgURE 3: State responses of second node.

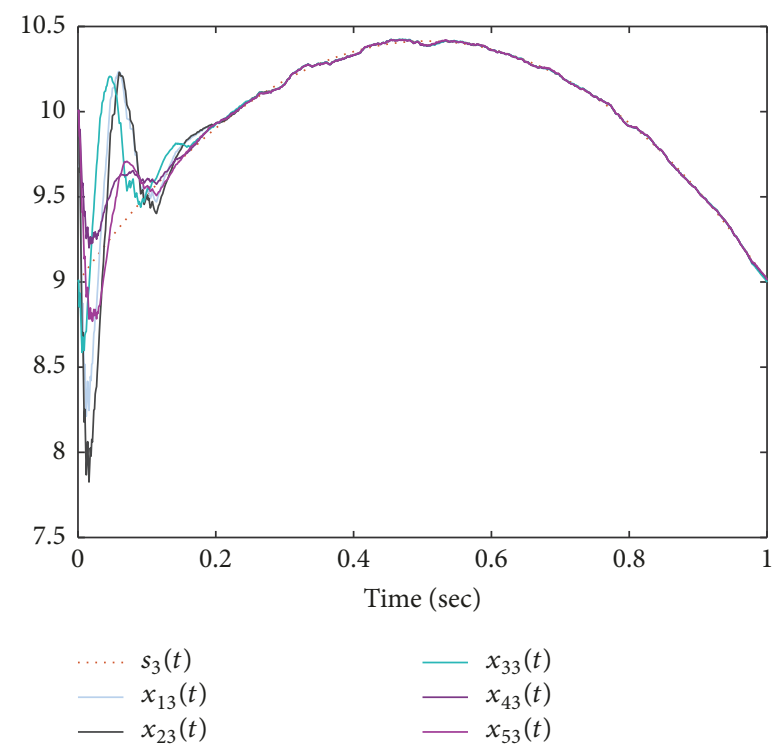

FIGURE 4: State responses of third node.

addition, to realize the finite-time synchronization, the time evolution of $e_{i}^{T}(t) Z_{p} e_{i}(t)(i=1,2,3,4,5),(p \in \mathbb{S})$ is depicted in Figure 12. It can be seen from Figure 12 that the states of the error system do not exceed the prescribed threshold $c_{2}=2$, which means that the synchronization of considered network (1) is achieved within a given finite-time interval. Thus, it can be concluded from the simulations that the designed nonfragile control algorithm effectively works even in the presence of stochastic noise and time-varying coupling delay.

\section{Conclusion}

In this paper, we have studied the robust finite-time nonfragile synchronization problem for a class of CDNs with

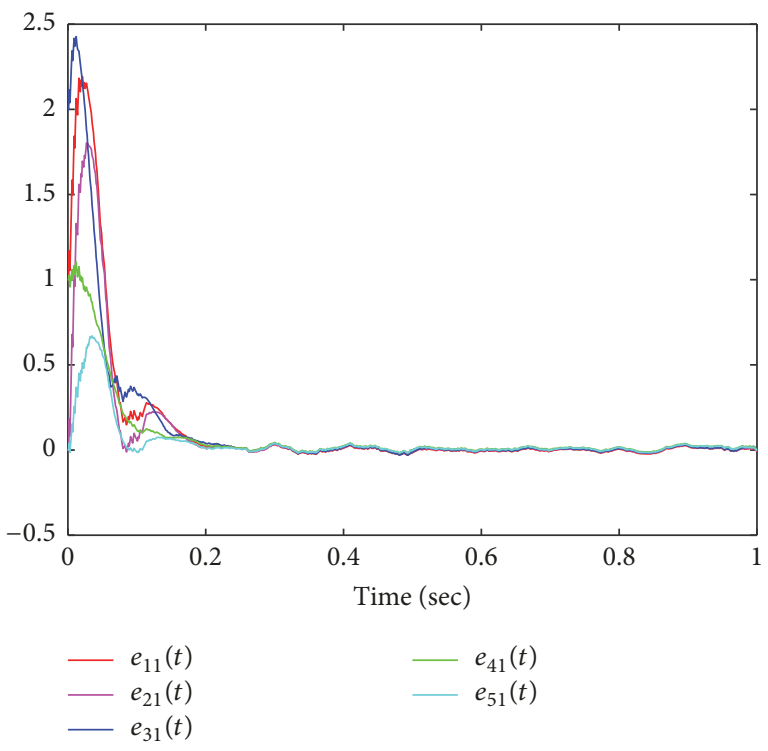

FIGURE 5: Error state responses of first node.

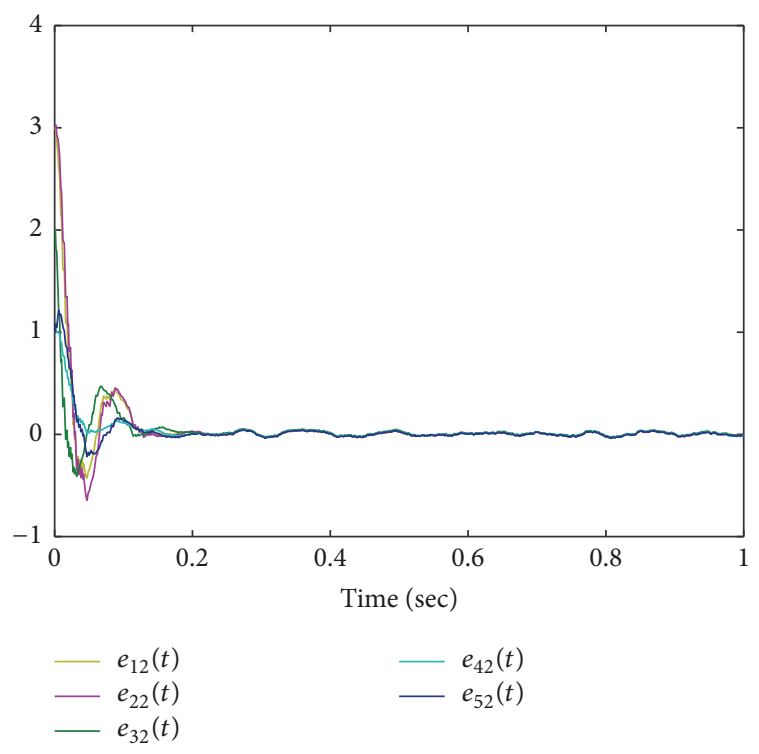

FIGURE 6: Error state responses of second node.

semi-Markov jump outer coupling, time-varying coupling delay, randomly occurring gain variation and stochastic noise. In particular, we have considered the semi-Markov switching topology to obtain the synchronization criterion. Moreover, we have introduced a stochastic variable satisfying the Bernoulli distribution to represent the random gain variations in the controller design. By employing the Lyapunov-Krasovskii stability theory and some stochastic analysis techniques, we then have developed a new finitetime stochastic synchronization criterion for the considered network in terms of linear matrix inequalities and have presented a design algorithm for the proposed nonfragile state feedback controller to a solution of the obtained set 


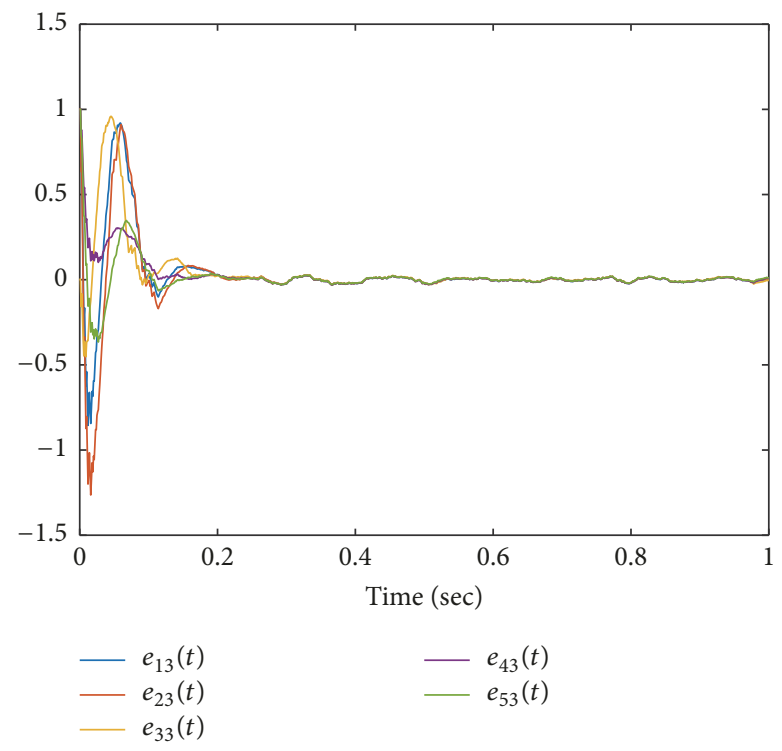

FIGURE 7: Error state responses of third node.

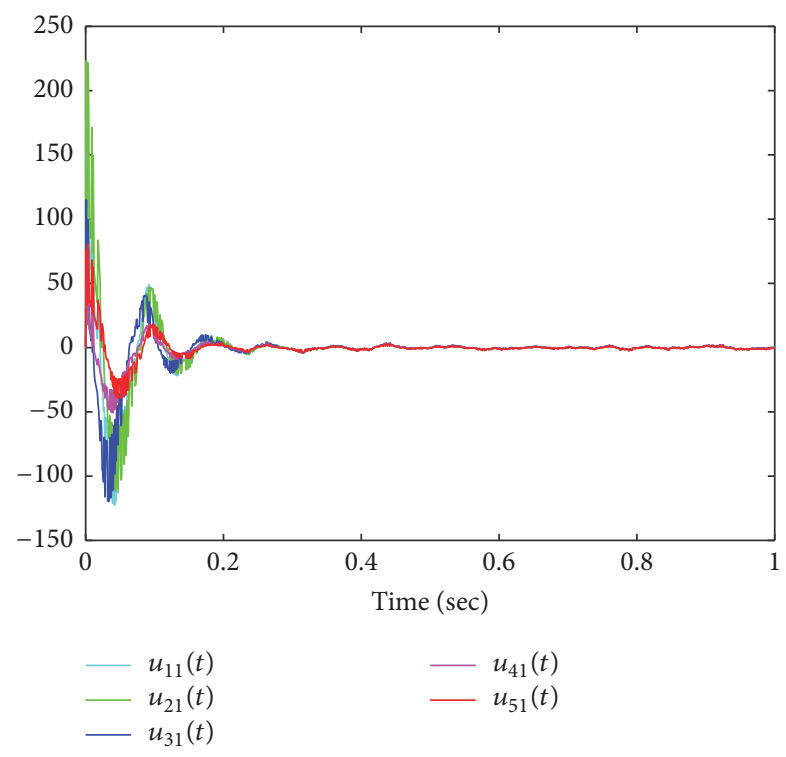

Figure 8: Control input of first node.

of linear matrix inequalities. At last, we have provided a numerical example to verify the obtained theoretical results. In addition, it should be pointed out that one of the future research topics would be to investigate the problem of finitetime mixed $H_{\infty}$ and passivity synchronization of stochastic singular CDNs with semi-Markov switching outer coupling delay and actuator saturation.

\section{Conflicts of Interest}

The authors declare that there are no conflicts of interest regarding the publication of this paper.

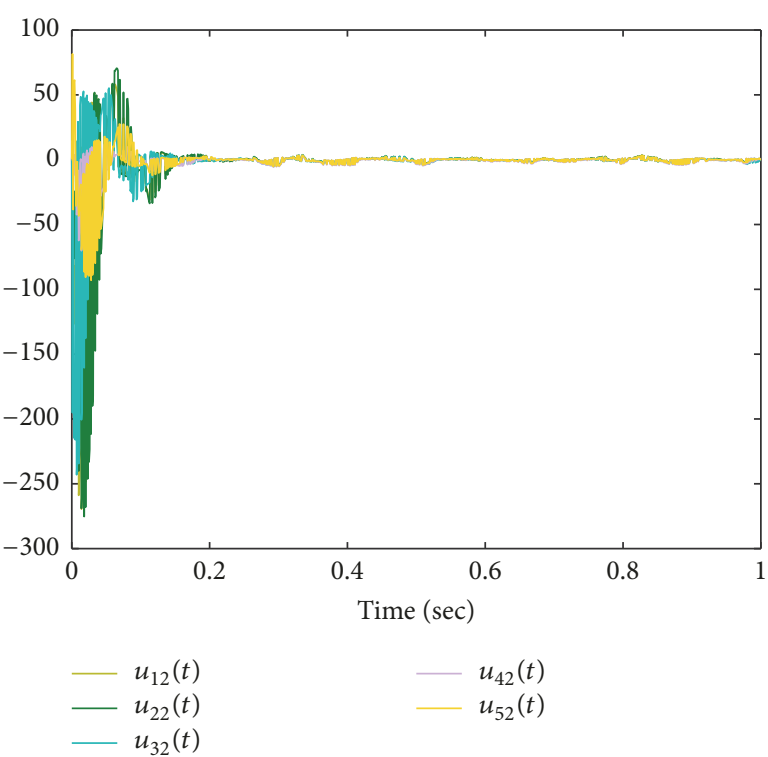

FIGURE 9: Control input of second node.

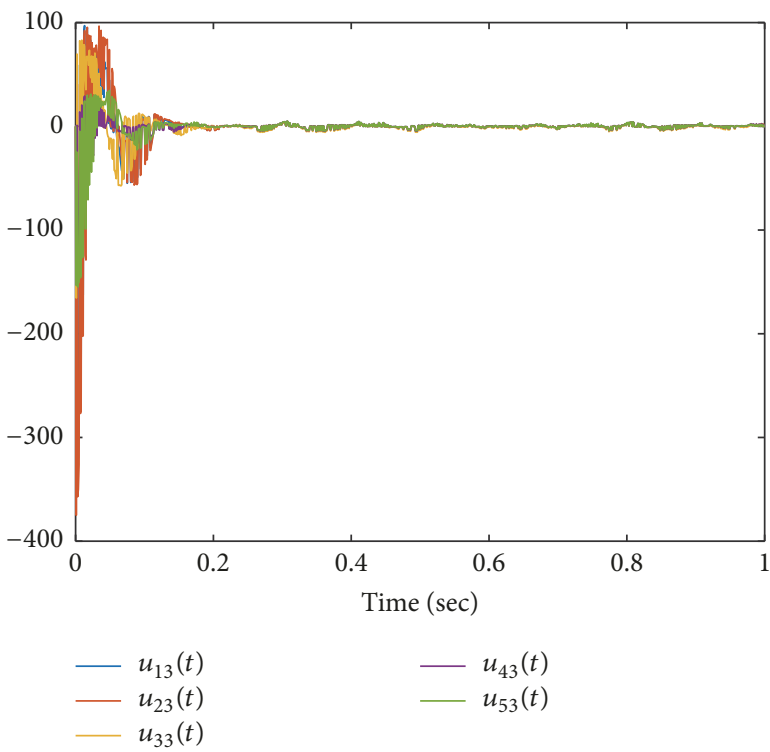

FIGURE 10: Control input of third node.

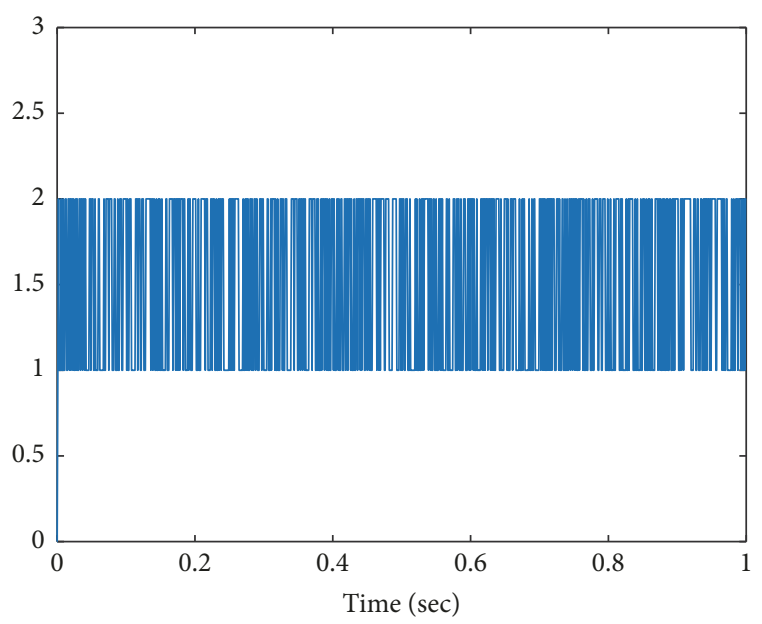

$\sigma(t)$

FIGURE 11: Jumping mode. 


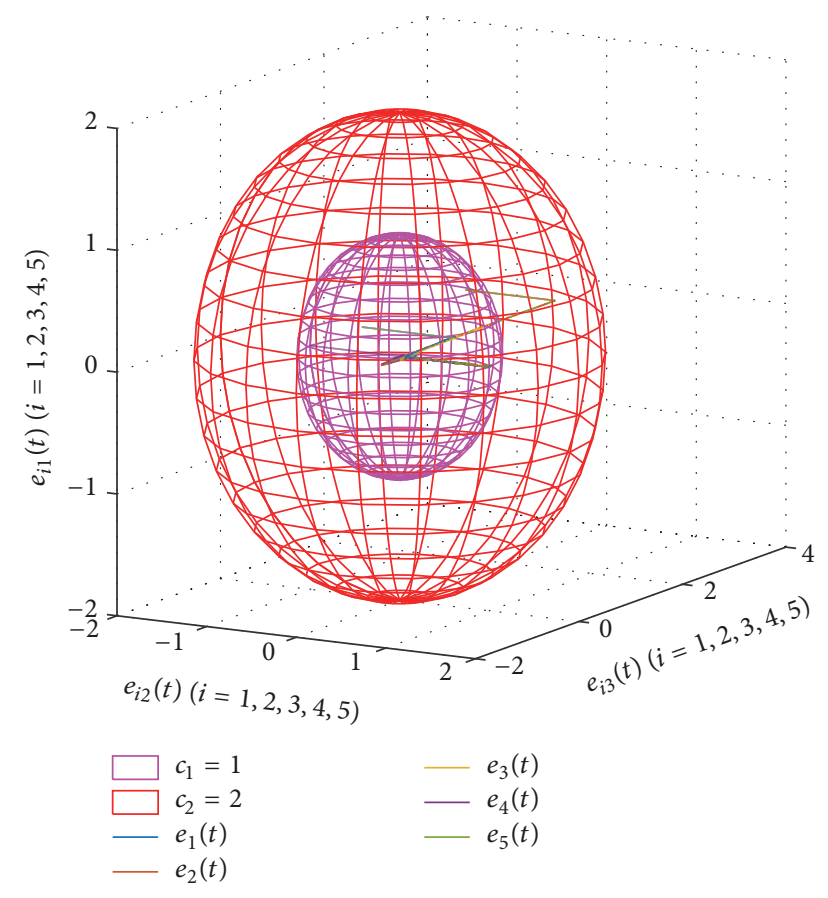

Figure 12: Evolution of $e_{i}^{T}(t) Z_{p} e_{i}(t)$.

\section{Acknowledgments}

The work of Yong-Ki Ma was supported by the National Research Foundation of Korea (NRF) grant funded by the Korean Government (MSIP) (no. 2015R1C1A1A01054663).

\section{References}

[1] R. Cohen and S. Havlin, "Complex networks: structure, robustness and function," Cambridge University Press, NY, USA, 2010.

[2] K. Aihara, J.-i. Imura, and T. Ueta, Analysis and Control of Complex Dynamical Systems, Springer, Tokyo, 2015.

[3] M. J. Park, O. M. Kwon, J. H. Park, S. M. Lee, and E. J. Cha, "Synchronization criteria of fuzzy complex dynamical networks with interval time-varying delays," Applied Mathematics and Computation, vol. 218, no. 23, pp. 11634-11647, 2012.

[4] T. H. Lee, J. H. Park, D. H. Ji, O. M. Kwon, and S. M. Lee, "Guaranteed cost synchronization of a complex dynamical network via dynamic feedback control," Applied Mathematics and Computation, vol. 218, no. 11, pp. 6469-6481, 2012.

[5] S. Cai, X. Lei, and Z. Liu, "Outer synchronization between two hybrid-coupled delayed dynamical networks via aperiodically adaptive intermittent pinning control," Complexity, vol. 21, no. S2, pp. 593-605, 2016.

[6] B. Kaviarasan, R. Sakthivel, and Y. Lim, "Synchronization of complex dynamical networks with uncertain inner coupling and successive delays based on passivity theory," Neurocomputing, vol. 186, 2015.

[7] J. Wang, L. Su, H. Shen, Z.-G. Wu, and J. H. Park, "Mixed $\mathrm{H} \infty$ /passive sampled-data synchronization control of complex dynamical networks with distributed coupling delay," Journal of The Franklin Institute, vol. 354, no. 3, pp. 1302-1320, 2017.
[8] X. Hao and J. Li, "Stochastic synchronization for complex dynamical networks with time-varying couplings," Nonlinear Dynamics, vol. 80, no. 3, pp. 1357-1363, 2015.

[9] G. He, J.-A. Fang, W. Zhang, and Z. Li, "Synchronization of switched complex dynamical networks with non-synchronized subnetworks and stochastic disturbances," Neurocomputing, vol. 171, pp. 39-47, 2016.

[10] R. Sakthivel, M. Sathishkumar, B. Kaviarasan, and S. Marshal Anthoni, "Synchronization and state estimation for stochastic complex networks with uncertain inner coupling," Neurocomputing, vol. 238, pp. 44-55, 2017.

[11] Z. Li, J.-a. Fang, T. Huang, and Q. Miao, "Synchronization of stochastic discrete-time complex networks with partial mixed impulsive effects," Journal of The Franklin Institute, vol. 354, no. 10, pp. 4196-4214, 2017.

[12] X.-J. Li and G.-H. Yang, "Graph theory-based pinning synchronization of stochastic complex dynamical networks," IEEE Transactions on Neural Networks and Learning Systems, vol. 28, no. 2, pp. 427-437, 2017.

[13] W.-H. Chen, Z. Jiang, X. Lu, and S. Luo, "Ho synchronization for complex dynamical networks with coupling delays using distributed impulsive control," Nonlinear Analysis: Hybrid Systems, vol. 17, pp. 111-127, 2015.

[14] J. Zhao, D. J. Hill, and T. Liu, "Synchronization of complex dynamical networks with switching topology: A switched system point of view," Automatica, vol. 45, no. 11, pp. 2502-2511, 2009.

[15] H. Shen, Y. Zhu, L. Zhang, and J. H. Park, "Extended dissipative state estimation for Markov jump neural networks with unreliable links," IEEE Transactions on Neural Networks and Learning Systems, vol. 28, no. 2, pp. 346-358, 2017.

[16] X. Song, Y. Men, J. Zhou, J. Zhao, and H. Shen, "Event-triggered Ho control for networked discrete-time Markov jump systems with repeated scalar nonlinearities," Applied Mathematics and Computation, vol. 298, pp. 123-132, 2017.

[17] Z.-X. Li, J. H. Park, and Z.-G. Wu, "Synchronization of complex networks with nonhomogeneous Markov jump topology," Nonlinear Dynamics, vol. 74, no. 1-2, pp. 65-75, 2013.

[18] H. Shen, Z.-G. Wu, Z. Zhang, and J. H. Park, "Non-fragile mixed Ho/l2-lo synchronisation control for complex networks with Markov jumping-switching topology under unreliable communication links," IET Control Theory \& Applications, vol. 8, no. 18, pp. 2207-2218, 2014.

[19] A. Wang, T. Dong, and X. Liao, "Event-triggered synchronization strategy for complex dynamical networks with the Markovian switching topologies," Neural Networks, vol. 74, pp. 52-57, 2016.

[20] H. Shen, Z.-G. Wu, and J. H. Park, "Reliable mixed passive and Ho filtering for semi-Markov jump systems with randomly occurring uncertainties and sensor failures," International Journal of Robust and Nonlinear Control, vol. 25, no. 17, pp. 32313251, 2015.

[21] F. Li, L. Wu, P. Shi, and C.-C. Lim, "State estimation and sliding mode control for semi-Markovian jump systems with mismatched uncertainties," Automatica, vol. 51, pp. 385-393, 2015.

[22] K. Liang, M. Dai, H. Shen, J. Wang, Z. Wang, and B. Chen, "L2-Lo synchronization for singularly perturbed complex networks with semi-Markov jump topology," Applied Mathematics and Computation, vol. 321, pp. 450-462, 2018.

[23] H. Shen, F. Li, S. Xu, and V. Sreeram, "Slow state variables feedback stabilization for semi-markov jump systems with singular 
perturbations," IEEE Transactions on Automatic Control, vol. pp, no. 99.

[24] H. Shen, L. Su, and J. H. Park, "Reliable mixed Ho/passive control for T-S fuzzy delayed systems based on a semi-Markov jump model approach," Fuzzy Sets and Systems, vol. 314, pp. 7998, 2017.

[25] T. H. Lee, Q. Ma, S. Xu, and J. H. Park, "Pinning control for cluster synchronisation of complex dynamical networks with semi-Markovian jump topology," International Journal of Control, vol. 88, no. 6, pp. 1223-1235, 2015.

[26] H. Shen, J. H. Park, Z.-G. Wu, and Z. Zhang, "Finite-time Ho synchronization for complex networks with semi-Markov jump topology," Communications in Nonlinear Science and Numerical Simulation, vol. 24, no. 1-3, pp. 40-51, 2015.

[27] Y. Zhang, Y. Shi, and P. Shi, "Robust and non-fragile finite-time Ho control for uncertain Markovian jump nonlinear systems," Applied Mathematics and Computation, vol. 279, pp. 125-138, 2016.

[28] Y. Zhang, Y. Shi, and P. Shi, "Resilient and robust finitetime Ho control for uncertain discrete-time jump nonlinear systems," Applied Mathematical Modelling: Simulation and Computation for Engineering and Environmental Systems, vol. 49, pp. 612-629, 2017.

[29] R. Sakthivel, S. Santra, B. Kaviarasan, and K. Venkatanareshbabu, "Dissipative analysis for network-based singular systems with non-fragile controller and event-triggered sampling scheme," Journal of The Franklin Institute, vol. 354, no. 12, pp. 4739-4761, 2017.

[30] W. Guan and F. Liu, "Non-fragile fuzzy dissipative static output feedback control for Markovian jump systems subject to actuator saturation," Neurocomputing, vol. 193, pp. 123-132, 2016.

[31] H. Shen, Y. Men, Z. Wu, and J. H. Park, "Nonfragile Ho control for fuzzy markovian jump systems under fast sampling singular perturbation," IEEE Transactions on Systems, Man, and Cybernetics: Systems, vol. pp, no. 99, pp. 1-12.

[32] Z.-G. Wu, J. H. Park, H. Su, and J. Chu, "Non-fragile synchronisation control for complex networks with missing data," International Journal of Control, vol. 86, no. 3, pp. 555-566, 2013.

[33] D. Li, Z. Wang, G. Ma, and C. Ma, "Non-fragile synchronization of dynamical networks with randomly occurring nonlinearities and controller gain fluctuations," Neurocomputing, vol. 168, pp. 719-725, 2015.

[34] G. Wang, L. Liu, Q. Zhang, and C. Yang, "Finite-time stability and stabilization of stochastic delayed jump systems via general controllers," Journal of The Franklin Institute, vol. 354, no. 2, pp. 938-966, 2017.

[35] X. Yang, Z. Wu, and J. Cao, "Finite-time synchronization of complex networks with nonidentical discontinuous nodes," Nonlinear Dynamics, vol. 73, no. 4, pp. 2313-2327, 2013.

[36] J. Mei, M. Jiang, W. Xu, and B. Wang, "Finite-time synchronization control of complex dynamical networks with time delay," Communications in Nonlinear Science and Numerical Simulation, vol. 18, no. 9, pp. 2462-2478, 2013.

[37] G. Mei, X. Wu, D. Ning, and J.-A. Lu, "Finite-time stabilization of complex dynamical networks via optimal control," Complexity, vol. 21, no. S1, pp. 417-425, 2016.

[38] L. Li, Z. Tu, J. Mei, and J. Jian, "Finite-time synchronization of complex delayed networks via intermittent control with multiple switched periods," Nonlinear Dynamics, vol. 85, no. 1, pp. 375-388, 2016. 


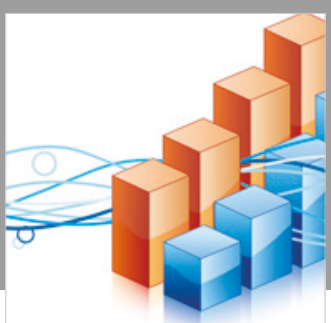

Advances in

Operations Research

\section{-n-m}
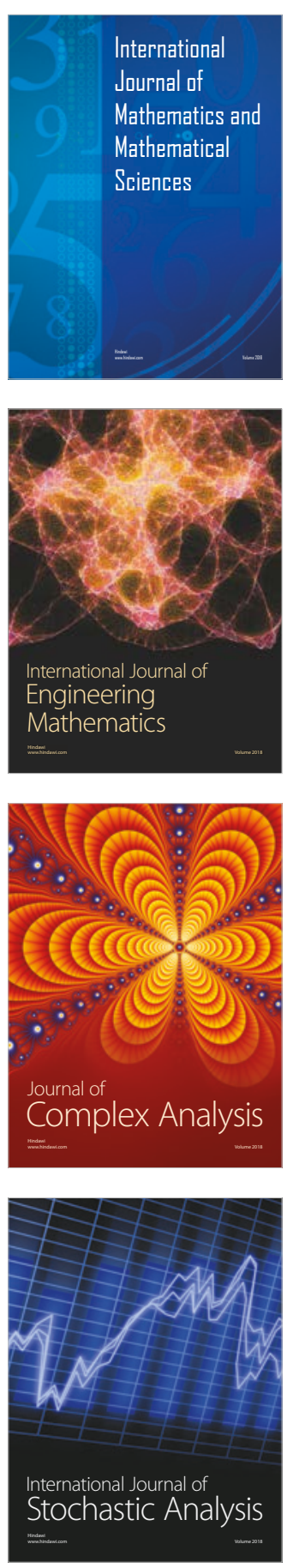
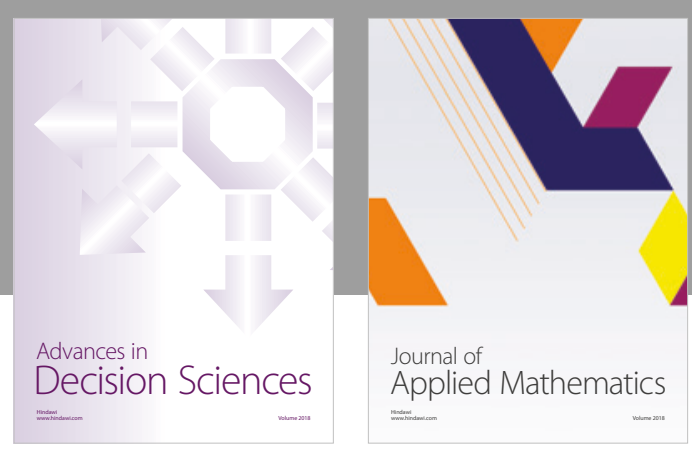

Journal of

Applied Mathematics
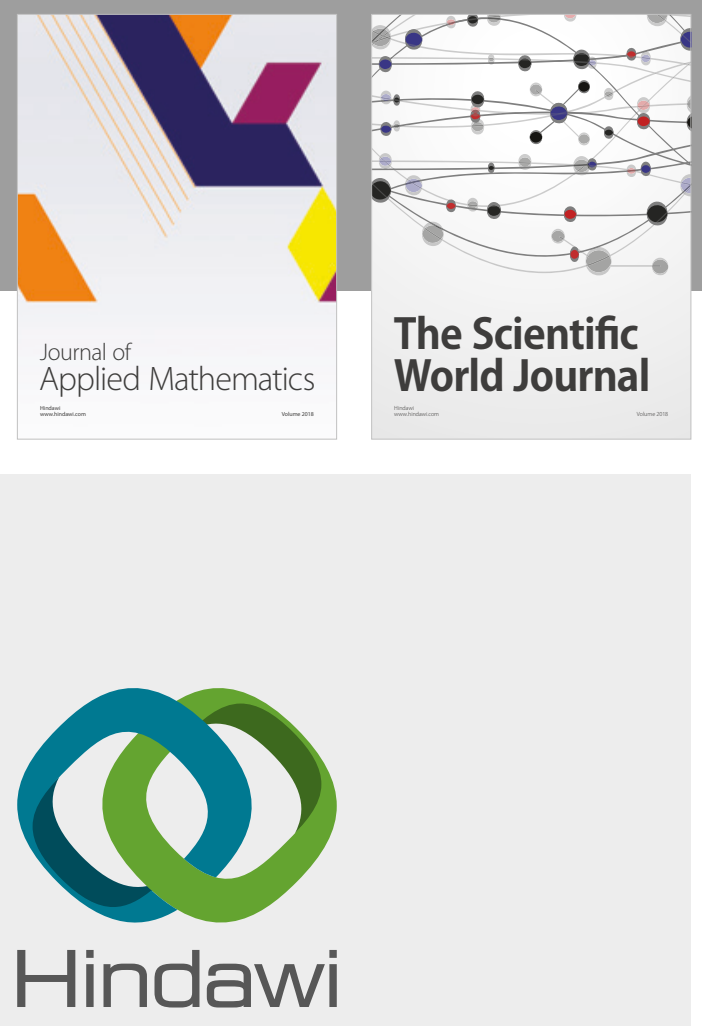

Submit your manuscripts at

www.hindawi.com

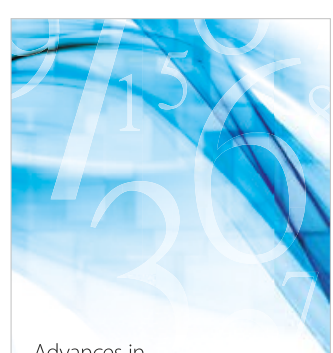

Advances in
Numerical Analysis
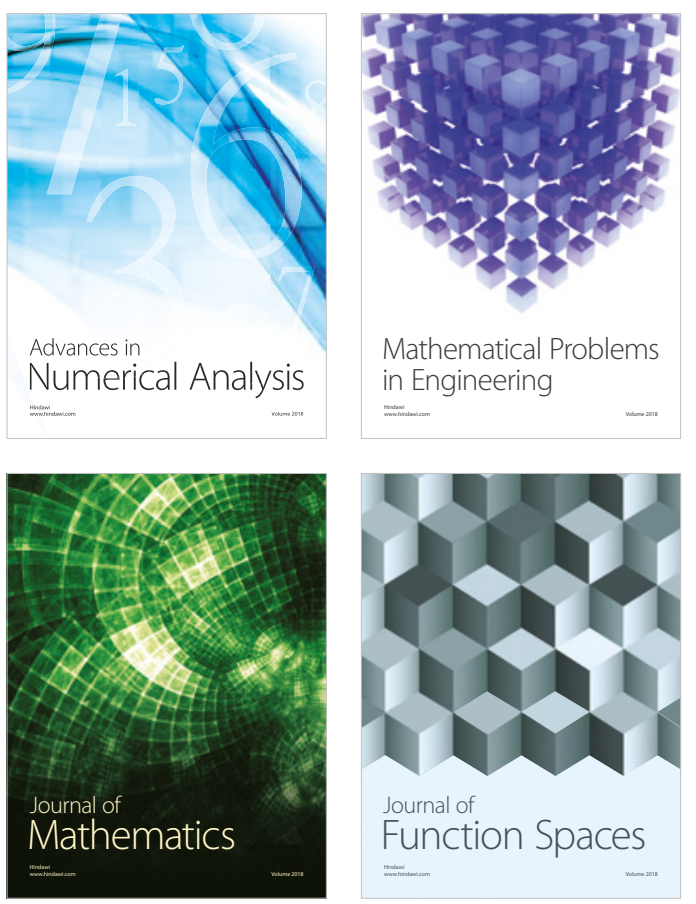

Mathematical Problems in Engineering

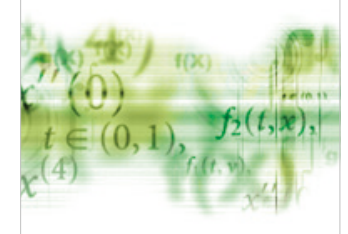

International Journal of

Differential Equations

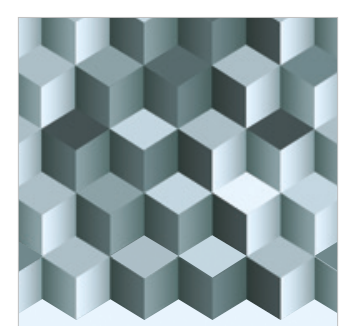

Journal of

Function Spaces
The Scientific

World Journal

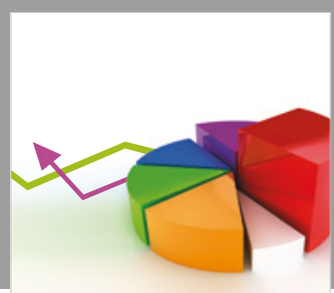

Journal of

Probability and Statistics
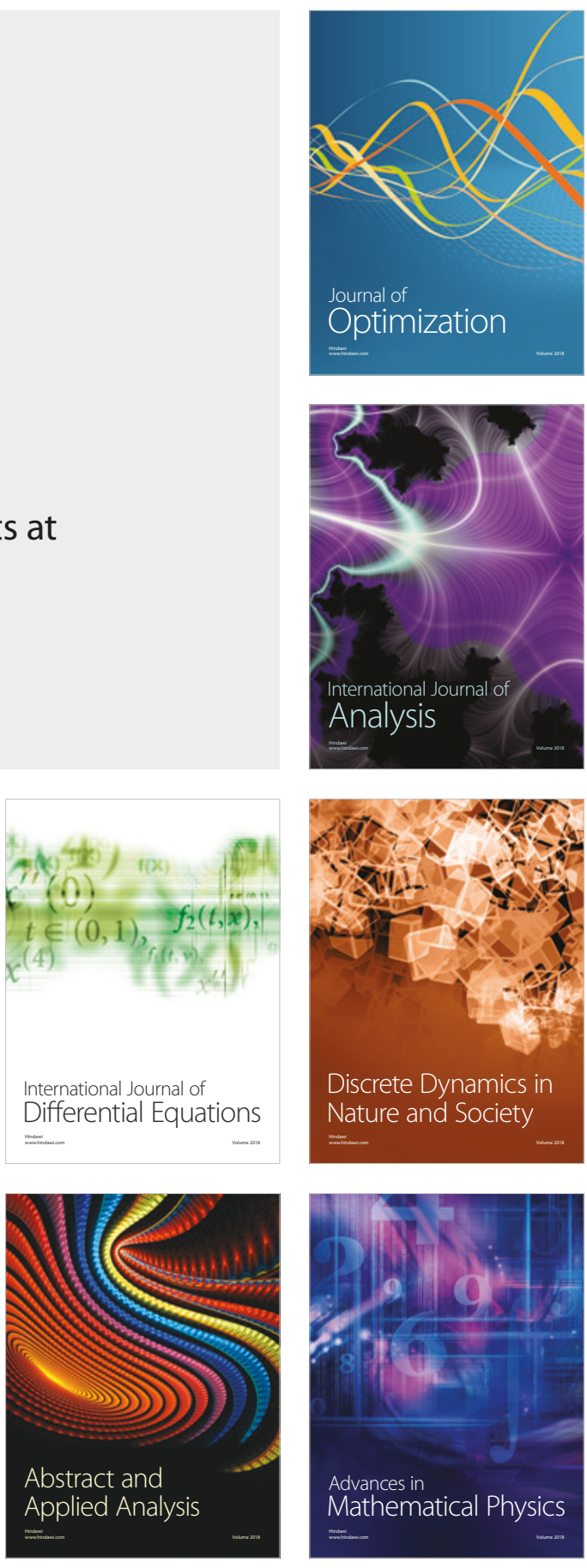